The influence of the Rashba spin-orbit coupling on the two-dimensional magnetoexcitons

This article has been downloaded from IOPscience. Please scroll down to see the full text article.

2011 J. Phys.: Condens. Matter 23345405

(http://iopscience.iop.org/0953-8984/23/34/345405)

View the table of contents for this issue, or go to the journal homepage for more

Download details:

IP Address: 139.179.139.67

The article was downloaded on 26/11/2012 at 15:56

Please note that terms and conditions apply. 


\title{
The influence of the Rashba spin-orbit coupling on the two-dimensional magnetoexcitons
}

\author{
T Hakioğlu ${ }^{1,2}$, M A Liberman ${ }^{3}$, S A Moskalenko ${ }^{4}$ and I V Podlesny ${ }^{4}$ \\ ${ }^{1}$ Department of Physics, Bilkent University, 06800 Ankara, Turkey \\ ${ }^{2}$ Institute of Theoretical and Applied Physics, 48740 Turunç, Muğla Turkey \\ ${ }^{3}$ Department of Physics, Uppsala University, Box 530, SE-751 21, Uppsala, Sweden \\ ${ }^{4}$ Institute of Applied Physics, Academy of Sciences of Moldova, 5, Academiei street, MD-2028, \\ Chisinau, Republic of Moldova
}

Received 6 May 2011, in final form 15 July 2011

Published 12 August 2011

Online at stacks.iop.org/JPhysCM/23/345405

\begin{abstract}
The influence of the Rashba spin-orbit coupling (RSOC) on the two-dimensional (2D) electrons and holes in a strong perpendicular magnetic field leads to different results for the Landau quantization in different spin projections. In the Landau gauge the unidimensional wave vector describing the free motion in one in-plane direction is the same for both spin projections, whereas the numbers of Landau quantization levels are different. For an electron in an s-type conduction band they differ by one, as was established earlier by Rashba (1960 Fiz. Tverd. Tela 2 1224), whereas for heavy holes in a p-type valence band influenced by the $2 \mathrm{D}$ symmetry of the layer they differ by three. The shifts and the rearrangements of the $2 \mathrm{D}$ hole Landau quantization levels on the energy scale are much larger in comparison with the case of conduction electron Landau levels. This is due to the strong influence of the magnetic field on the RSOC parameter. At sufficiently large values of this parameter the shifts and rearrangements are comparable with the hole cyclotron energy. There are two lowest spin-split Landau levels for electrons as well as four lowest ones for holes in the case of small RSOC parameters. They give rise to eight lowest energy bands of the $2 \mathrm{D}$ magnetoexcitons, as well as of the band-to-band quantum transitions. It is shown that three of them are dipole-active, three are quadrupole-active and two are forbidden. The optical orientation under the influence of circularly polarized light leads to optical alignment of the magnetoexcitons with different orbital momentum projections in the direction of the external magnetic field.
\end{abstract}

(Some figures in this article are in colour only in the electronic version)

\section{Introduction}

The influence of the spin-orbit coupling (SOC) on the two-dimensional (2D) Wannier-Mott excitons in double quantum well (DQW) structures, as well as the possibilities of nonconventional electron-hole $(\mathrm{e}-\mathrm{h})$ pairing in these conditions were discussed in [2,3]. The main results are breaking of the spin degeneracy of the electrons and holes, changes of the exciton structure, and new properties of the Bose-Einstein condensed excitons. There are two types of SOC. One of them described by Dresselhaus [4] is known to be intrinsically present in zinc blende structure. The Rashba spin-orbit coupling (RSOC) [1,5] depends on the electric field strength $E_{z}$ perpendicular to the layer surface.

Side by side with questions related to the Bose-Einstein condensation (BEC) phenomenon, there exist a vast number of investigations in the field of spin-orbit coupling effects. Some of them will be cited below, including the special, instructive monograph [6] and the papers [7-13]. Since the mid 1980s, as was mentioned in [7], there has been extensive interest in the effects of an applied electric field normal to the layers on the optical properties of semiconductor quantum wells (QWs) and superlattices (SLs). The arising inversion asymmetry leads to anisotropic optical transitions. 
The theoretical calculations of the Pockels effect for GaAs/ $\mathrm{Ga}_{1-x} \mathrm{Al}_{x} \mathrm{As}$ and SLs demonstrate this statement [7]. In [6] it was underlined that the spin degeneracy of the electron and hole states is the combined effect of the inversion symmetry in space and time. The first operator we denote by $\hat{I}$ and the second one by $K=\sigma_{2} K_{0}$, where $\sigma_{2}$ is the Pauli matrix and $K_{0}$ is the complex conjugation operation. They change the Bloch wavefunctions in solids characterized by the wavevector $\vec{k}$ and spin index $\sigma$ with two projections in the following way:

$$
\begin{aligned}
I \Psi_{\sigma}(\vec{r}, \vec{k})=\Psi_{\sigma}(-\vec{r}, \vec{k}) & =\Psi_{\sigma}(\vec{r},-\vec{k}), & & \sigma=\uparrow, \downarrow \\
\hat{K} \Psi_{\uparrow}(\vec{r}, \vec{k})=\Psi_{\downarrow}^{*}(\vec{r}, \vec{k}) & =\Psi_{\downarrow}(\vec{r},-\vec{k}), & & \hat{K}^{2}=-1 .
\end{aligned}
$$

The time inversion operator $\hat{K}$ flips the spin side by side with the complex conjugation operation.

The first symmetry operator gives the equality

$$
E_{\sigma}(\vec{k})=E_{\sigma}(-\vec{k})
$$

whereas the time inversion operator leads to Kramers degeneracy

$$
E_{\uparrow}(\vec{k})=E_{\downarrow}(-\vec{k}) .
$$

It takes place, even if the space inversion is absent. The invariance of the Hamiltonian under the action of two inversion operations leads to two-fold spin degeneracy of the single-particle states with arbitrary wavevector $\vec{k}$ as follows:

$$
E_{\uparrow}(\vec{k})=E_{\uparrow}(-\vec{k})=E_{\downarrow}(-\vec{k}) ; \quad E_{\uparrow}(\vec{k})=E_{\downarrow}(\vec{k}) .
$$

These relations are true for both electrons and holes. Nevertheless the Rashba spin splitting of 2D hole systems is very different from the more familiar case of $2 \mathrm{D}$ electron systems. In [6] this was explained by the fact that the holes have typically larger masses and smaller kinetic energies. The SOC is more important for holes than for electrons. When the carriers are moving through the inversion asymmetric potential, the spin degeneracy is removed even in the absence of an external magnetic field $B$. In this case there are two different branches of energy, $E_{\uparrow}(\vec{k}) \neq E_{\downarrow}(\vec{k})$, and the spin splitting is present. In quasi-2D QWs this spin splitting can be the consequence of a bulk inversion asymmetry (BIA) of the underlying crystal (for example, as in zinc blende crystals), or of a structure inversion asymmetry (SIA) of the confinement potential.

In both cases of inversion asymmetry the spin splitting takes place in the absence of $B$, i.e. $E_{\uparrow}(\vec{k}) \neq E_{\downarrow}(\vec{k})$, but the Kramers degeneracy continues to exist, $E_{\uparrow}(\vec{k})=E_{\downarrow}(-\vec{k})$. This spin splitting is not due to the Zeeman effect because $B=0$. In [6] the origin of the spin splitting is related with the motion of the electron through the inversion asymmetric spatial environment, the interaction with which is due to the SOC. The periodic parts of the electron Bloch functions are affected by the atomic fields that enter into the Pauli spin-orbit term, whereas the envelope functions are affected by the macroscopic environment. Following this picture, SIA leads to spin splitting, which is due to both the macroscopic electric field and the microscopic electric field from the atomic cores.
SIA spin splitting is always proportional to the macroscopic field strength times a prefactor depending on the microscopic spin-orbit interaction (SOI). This prefactor depends only on the matrix elements of the microscopic SOI and is due completely to the BIA. To reveal the origin of the spin splitting in a simpler way the following idea was suggested. One can imagine the electron moving with velocity $V_{\|}$to the plane of the layer subjected to the action of a perpendicular electric field $E_{z}$.

In the reference frame moving together with the electron the Lorentz transformation induces the magnetic field $B=$ $\left(V_{\|} / c\right) E_{z}$, which acts on the electron spin giving rise to such an indirect Zeeman effect. The estimations made showed that the spin splitting obtained in such a way is 5-6 orders of magnitude smaller than the experimentally observed values of the SOC. The discrepancy is due to the fact that the idea of Lorentz transformation neglects the contribution of the atomic cores to the SOI felt by Bloch electrons in a solid [6]. Another important detail, which must be remembered, is related to the crystallographic symmetry group of the solids. The spin splitting induced by the atomic cores, which is also called BIA splitting, also depends on the irreducible representations of the double group of the wavevector $\vec{k}$. For example, in the case of $\vec{k}$ parallel to the $\langle 111\rangle$ direction the wavevector group is $\mathrm{C}_{3 \mathrm{v}}$. It has the double-group irreducible representations $\Gamma_{4}, \Gamma_{5}$ and $\Gamma_{6}$. In the case when the electron and light hole (LH) states transform according to the 2D representations $\Gamma_{4}$, whereas the heavy hole $(\mathrm{HH})$ states transform according to the $1 \mathrm{D}$ representations $\Gamma_{5}$ and $\Gamma_{6}$, the BIA spin splitting vanishes for electrons and LHs and exists for HHs [6]. RSOC and intrinsic SOI under certain conditions lead to a Dirac cone formation out of a parabolic band and it is possible to create a 'Mexican-hat-like' energy dispersion law [8]. The Mexican-hat-like dispersion has a line of degenerate low-energy points forming a ring. It can appear in a variety of physical systems. Such peculiarities were demonstrated in $[2,3]$. The Mexican-hat-like dispersion law leads to a weak crystallization transition [9], whereas in cold atom physics it gives rise to topologically different ground states of the Bose-Einstein condensed atoms and molecules [8].

Now the Landau quantization of electrons and holes depending on their band structure will be discussed. In the calculations of $[10,11]$ of the hole Landau levels in the strained asymmetric p-type $\mathrm{GeSi}_{x} \mathrm{Ge}_{1-x}$ QWs the cyclotron masses were determined. Self-consistent hole subband calculations were combined with calculations of the Landau levels using a $6 \times 6 \vec{k} \cdot \vec{p}$ Hamiltonian for the topmost $\Gamma_{8}$ and $\Gamma_{7}$ bulk valence bands.

To include the magnetic field in the calculation of the Landau levels the canonical momentum $\hat{p}=-\mathrm{i} \hbar \vec{\nabla}$ is substituted by the kinetic momentum $\hat{\pi}=\hat{p}-\frac{q}{c} \vec{A}(\vec{r})$, where $q$ is the electric charge of the quasi-particle [6]. These questions will be discussed in section 2 .

As was mentioned in $[12,13]$ the Rashba model can be described by purely group theoretical means. For an electron in an s-like conduction band the total angular momentum with SOI is $j=1 / 2$. Both the wavevectors $\vec{k}$ and the electric strength $\vec{E}$ are polar vectors, whereas their cross product 
$[\vec{k} \times \vec{E}]$ is an axial vector. Its point product with the spin axial vector $\vec{\sigma}$ gives rise to the triple scalar product $[\vec{k} \times \vec{E}] \cdot \vec{\sigma}$. This expression is an invariant under the action of the group symmetry elements forming the identity representation $\Gamma_{1}$. Similar arguments were given in [14]. In the first quantization representation the wavevector $\vec{k}$ is substituted by $-\mathrm{i} \vec{\nabla}$. In the $\Gamma_{6}$-type conduction band the triple scalar product is the only term of the first order in $\vec{\nabla}$ and $\vec{E}$ compatible with the symmetry of the band. RSOC

The band structure described by the Hamiltonian with

$$
\begin{gathered}
H_{\mathrm{e}}=-\frac{\hbar^{2} \triangle_{\|}}{2 m_{\mathrm{e}}} \hat{I}-\mathrm{i} \alpha\left(\hat{\sigma}_{x} \frac{\partial}{\partial y}-\hat{\sigma}_{y} \frac{\partial}{\partial x}\right) ; \\
\Delta_{\|}=\frac{\partial^{2}}{\partial x^{2}}+\frac{\partial^{2}}{\partial y^{2}} ; \quad \alpha=\alpha_{\mathrm{e}} E_{z}
\end{gathered}
$$

has the dispersion laws

$$
E_{\mathrm{e}}^{ \pm}=\frac{\hbar^{2} k_{\|}^{2}}{2 m_{\mathrm{e}}} \pm|\alpha| k_{\|} ; \quad k_{\|}=\sqrt{k_{x}^{2}+k_{y}^{2}} .
$$

One of them contains the loop of minima $[1,5]$. The topmost valence band in our case is p-like with orbital quantum number $l=1$ and with total angular momentum equal to $j=3 / 2$. The four-fold band states give rise to HHs and LHs forming in cubic crystals the irreducible representation $\Gamma_{8}$ at the point $k=0$. The angular momentum matrices with $j=3 / 2$ give rise to axial vectors $\vec{J}=\left(J_{x}, J_{y}, J_{z}\right)$ and $\overrightarrow{\mathcal{J}}=\left(J_{x}^{3}, J_{y}^{3}, J_{z}^{3}\right)$.

Now the SOI Hamiltonian contains two invariants

$$
H_{\mathrm{h}}^{\mathrm{SOC}}=\beta_{1}[\vec{k} \times \vec{E}] \cdot \vec{J}+\beta_{2}[\vec{k} \times \vec{E}] \cdot \overrightarrow{\mathcal{J}} .
$$

In $3 \mathrm{D}$ structures the prefactor $\beta_{2}$ is always much smaller than $\beta_{1}[12,13]$, but in $2 \mathrm{D}$ structures the $\Gamma_{8}$ four-fold band is split into HHs and LHs [21].

For the LHs the effective Rashba Hamiltonian has the lowest order in the $\vec{k}$ term and is the same as for the conduction electrons. For the HHs the effective Rashba Hamiltonian happens to be third order in $\vec{k}$ and remains first order in the spin operators $\vec{\sigma}$ as follows [12, 13]:

$$
H_{\mathrm{h}}^{\mathrm{SOC}}=\beta_{\mathrm{h}} E_{z}\left(\hat{\sigma}_{+} k_{-}^{3}-\hat{\sigma}_{-} k_{+}^{3}\right)
$$

where

$$
\begin{array}{ccc}
\sigma_{ \pm}=\frac{1}{2}\left(\hat{\sigma}_{x} \pm \mathrm{i} \hat{\sigma}_{y}\right) ; & k_{ \pm}=\left(k_{x} \pm \mathrm{i} k_{y}\right) ; \\
\hat{\sigma}_{x}=\left|\begin{array}{cc}
0 & 1 \\
1 & 0
\end{array}\right| ; & \hat{\sigma}_{y}=\left|\begin{array}{cc}
0 & -\mathrm{i} \\
\mathrm{i} & 0
\end{array}\right| ; \\
\hat{\sigma}_{z}=\left|\begin{array}{cc}
1 & 0 \\
0 & -1
\end{array}\right| ; & \hat{I}=\left|\begin{array}{cc}
1 & 0 \\
0 & 1
\end{array}\right| .
\end{array}
$$

The Hamiltonians (5) and (8) contain chirality terms of the type $\left(K_{ \pm}\right)^{J}$. As is usual in the literature the values $J=1-3$ are considered. Two of them, $J=1$ and 3, are the topics of our paper. The first order chirality $(J=1)$ discussed by Rashba [1] and Dresselhaus [4] in 3D crystals also occurs in single layer graphene [15-17]. Third order chirality occurs for HHs in GaAs-type QWs with the 2D crystal group of symmetry. This was well grounded in the text of $[12,13]$.
Second order chirality is realized in biased and unbiased bilayer graphene as one can see from [18-20]. The massless Dirac-type fermions in single layer graphene moving along the cyclotron trajectory accumulate a Berry phase equal to $\pi$, whereas the fermions in bilayer graphene accumulate the phase $2 \pi$. This difference influences the quantum Hall effect taking place in these structures [20]. Alongside a single term $\left(K_{ \pm}\right)^{J}$ one can consider also the mixed combinations.

Now we will add the zero order Hamiltonian supposing that the holes have the same parabolic dispersion law $\hbar^{2} k_{\|}^{2} / 2 m_{\mathrm{h}}$ as the conduction electrons, but with different mass. Below we will see that in the presence of the RSOC such a simple supposition is not sufficient. Indeed, the parabolic Hamiltonian together with the RSOC Hamiltonian (8) gives rise to two spinor branches of the complete dispersion law [12, 22]

$$
E_{\mathrm{h}}^{ \pm}\left(k_{\|}\right)=\frac{\hbar^{2} \vec{k}_{\|}^{2}}{2 m_{\mathrm{h}}} \pm\left|\frac{\beta_{\mathrm{h}} E_{z}}{2}\right|\left|\vec{k}_{\|}\right|^{3} .
$$

The lower branch with the minus sign due to the term $k_{\|}^{3}$ has an unlimited decrease penetrating deeply inside the energy gap at large values of $k_{\|}$. To avoid such an unphysical situation we will add into the zero order Hamiltonian a positive quartic term $\left|\delta_{\mathrm{h}} E_{z}\right| k_{\|}^{4}$ with the positive parameter $\left|\delta_{\mathrm{h}} E_{z}\right|$ also proportional to $E_{z}$ as the cubic term; the parameter $\delta_{\mathrm{h}}$ can be determined experimentally. This correction leads to the new dispersion law

$$
\frac{\hbar^{2} k_{\|}^{2}}{2 m_{\mathrm{h}}}-\left|\frac{\beta_{\mathrm{h}} E_{z}}{2}\right| k_{\|}^{3}+\left|\delta_{\mathrm{h}} E_{z}\right| k_{\|}^{4}, \quad\left|\delta_{\mathrm{h}} E_{z}\right|>0 .
$$

It only has a minimum at the point $k_{\|}=0$ if the term $\left|\beta_{\mathrm{h}} E_{z} / 2\right|$ is small and supplementary extrema, one maximum and a second minimum, if the parameter $\left|\beta_{\mathrm{h}} E_{z}\right|^{2}$ exceeds some value

$$
\left|\beta_{\mathrm{h}} E_{z}\right|^{2} \geq \frac{64}{9} \frac{\hbar^{2}\left|\delta_{\mathrm{h}} E_{z}\right|}{m_{\mathrm{h}}} ; \quad\left|E_{z}\right| \geq \frac{64}{9} \frac{\hbar^{2}\left|\delta_{\mathrm{h}}\right|}{m_{\mathrm{h}} \beta_{\mathrm{h}}^{2}} .
$$

In the case of equality the last two extrema merge into a bending point $k_{\|}=3\left|\beta_{\mathrm{h}}\right| /\left(16\left|\delta_{\mathrm{h}}\right|\right)$. In the case of a conduction electron the RSOC term is proportional to $\left|\vec{k}_{\|}\right|$instead of $\left|\vec{k}_{\|}\right|^{3}$ and the lower dispersion branch has a loop of minima as Rashba had shown $[1,5]$ without deep penetration into the energy gap. These properties of the spinor dispersion laws for electrons and holes are represented in figure 1. The energies for electrons and holes are counted in opposite directions. They will be taken into account in the process of Landau quantization.

The electric field strength $E_{z}$ depends on the density of charges in the system $[12,13]$. The interaction constants $\alpha_{\mathrm{e}} E_{z}$ and $\beta_{\mathrm{h}} E_{z}$ were evaluated in $[2,3]$ for different values of $E_{z}$, arriving at the conclusion that at $E_{z}=100-200 \mathrm{kV} \mathrm{cm}^{-1}$ the RSOC is a dominant mechanism for the energy band spin splitting. The main goals of the $[2,3]$ were to show that changing these parameters is an alternative method to examine the BEC of the 2D Wannier-Mott excitons and their crossover from the low density regime with atom-like structure of the $\mathrm{e}-\mathrm{h}$ pairs to the high density $\mathrm{e}-\mathrm{h}$ pairs. In the latter 

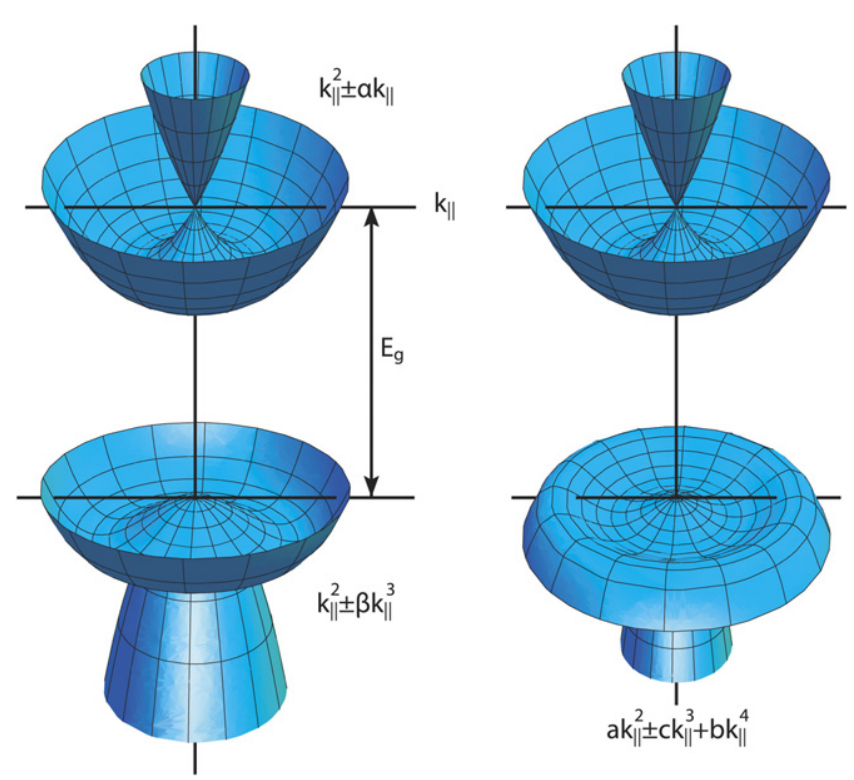

Figure 1. The dispersion laws for the $2 \mathrm{D}$ e-h pair. The upper dispersion laws concern the conduction electron and follow the formula (6). The lower dispersion laws concern the 2D HHs. The left part is drawn following [12] and the formula (10), whereas the right part reflects the expression (11). The energies for the electrons and holes are counted in opposite directions.

case the electrons and holes form a Fermi degenerate gas. The weak interaction of the particles near the corresponding Fermi levels gives rise to their coherent pairing and to Bardeen-Cooper-Schrieffer (BCS)-type condensation [23].

In the presence of the SOC the real excitonic order parameter is changed due to the mixing of the spin states. One of the most distinct effects, which is expected to take place in the frame of exciton condensation is the controllable mixing of the dark and bright exciton states [2,3]. Their mixing could lead to a change of the intensity of the coherent light emission. It was supposed that the ground exciton state is composed predominantly of the dark excitons, which do not couple to the light due to the total spin projections of the e-h pair being equal to \pm 2 . In the frame of this model the bright excitons with spins $( \pm 1)$ are situated above the dark excitons. Similar questions will be discussed below in the case of $2 \mathrm{D}$ magnetoexcitons.

This paper is organized as follows. In section 2 following the papers [1,5] we will apply the Landau quantization procedure in the variant of the SOC Hamiltonian (8) depending on the wavevector $k_{ \pm}^{3}$. The SOI couples the Landau levels corresponding to one spin projection with the Landau levels related to another spin projection. The numbers of these correlated Landau levels differ by one when the SOC Hamiltonian is expressed by the first order wavevector $\vec{k}$ and they differ by three when the SOC Hamiltonian contains the third order components $k_{ \pm}^{3}$ of the wavevector. We believe that the latter variant represents the original results of the second section. Section 3 is dedicated to calculations of the matrix elements of the Coulomb interaction between electrons and holes on the basis of the new spin-split states. The energy spectrum of the 2D magnetoexcitons with mixed spin orientations will be derived. The quantum transitions from the ground state of the crystal to the new exciton states will be described in section 4 paying most attention to the mixing of the bright and dark states.

\section{The Landau quantization of two-dimensional holes in the presence of Rashba spin-orbit coupling}

Following the papers [1-3, 5, 12, 13] the full Hamiltonian describing the Landau quantization of 2D holes in a strong perpendicular magnetic field taking into account the RSOC consists of two parts. The zero order Hamiltonian for holes is

$H_{0}=\left[\frac{1}{2 m_{\mathrm{h}}}\left(\hat{\vec{P}}-\frac{|e|}{c} \vec{A}(\vec{r})\right)^{2}+\left|\delta_{\mathrm{h}} E_{z}\right|\left(\hat{\vec{P}}-\frac{|e|}{c} \vec{A}(\vec{r})\right)^{4}\right] \vec{I}$,

where $\hat{\vec{P}}=\hbar \hat{k}=-\mathrm{i} \hbar \vec{\nabla}$ is the canonical momentum and the vector potential is written in the Landau gauge $\vec{A}(\vec{r})=\left(A_{x}=\right.$ $-H y, A_{y}=0, A_{z}=0$ ).

The second part of the full Hamiltonian is the RSOC Hamiltonian (8), in which instead of the canonical momentum, the kinetic momentum is introduced. It has the properties

$$
\begin{array}{cc}
\hbar \hat{K}=\hat{P}-\frac{|e|}{c} \vec{A}(\vec{r}) ; & \hat{K}=\hat{k}-\frac{|e|}{c \hbar} \vec{A}(\vec{r}) ; \\
\hat{K}_{x}=-\mathrm{i} \frac{\partial}{\partial x}+\frac{y}{l^{2}} ; & \hat{K}_{y}=-\mathrm{i} \frac{\partial}{\partial y} ; \\
{\left[\hat{K}_{x}, \hat{K}_{y}\right]=\frac{\mathrm{i}}{l^{2}}} & K_{ \pm}=K_{x} \pm \mathrm{i} K_{y} ; \\
{\left[K_{+}, K_{-}\right]=\frac{2}{l^{2}} ;} & l^{2}=\frac{\hbar c}{|e| H} .
\end{array}
$$

Following [24-28] we will introduce the creation and annihilation operators for the holes with positive electric charge $\operatorname{sgn}(q)=+1, q=|e|$ in the form

$$
a=\frac{l}{\sqrt{2}} K_{+}, \quad a^{\dagger}=\frac{l}{\sqrt{2}} K_{-} ; \quad\left[a, a^{\dagger}\right]=1 .
$$

Indeed, acting with these operators in differential form on the wavefunctions of the Landau quantization, which in real space representation and Landau gauge have the expressions $\left(\exp [\mathrm{i} g x] / \sqrt{L_{x}}\right) \varphi_{n}(y)$, one can obtain

$$
\begin{gathered}
a^{\dagger} \frac{\mathrm{e}^{\mathrm{i} g x}}{\sqrt{L_{x}}} \varphi_{n}(y)=\frac{\mathrm{e}^{\mathrm{i} g x}}{\sqrt{L_{x}}} \frac{1}{\sqrt{2}}\left(\eta-\frac{\partial}{\partial \eta}\right) \varphi_{n}(\eta), \\
a \frac{\mathrm{e}^{\mathrm{i} g x}}{\sqrt{L_{x}}} \varphi_{n}(y)=\frac{\mathrm{e}^{\mathrm{i} g x}}{\sqrt{L_{x}}} \frac{1}{\sqrt{2}}\left(\eta+\frac{\partial}{\partial \eta}\right) \varphi_{n}(\eta), \\
\eta=g l+l \frac{\partial}{\partial y} .
\end{gathered}
$$

The increasing and decreasing differential operators $\frac{1}{\sqrt{2}}(\eta-$ $\left.\frac{\partial}{\partial \eta}\right)$ and $\frac{1}{\sqrt{2}}\left(\eta+\frac{\partial}{\partial \eta}\right)$ in the space of wavefunctions $\varphi_{n}(\eta)$ play the same role as the Bose operators $a^{\dagger}, a$ in the space of the Fock states $|n\rangle=\frac{\left(a^{\dagger}\right)^{n}}{\sqrt{n !}}|0\rangle$ in the second quantization 
representation. In this representation using the Bose operators $a^{\dagger}, a$, the zero order Hamiltonian (13) can be written

$$
\begin{aligned}
& H_{0}=\hbar \omega_{\mathrm{ch}}\left[\left(a^{\dagger} a+\frac{1}{2}\right)+\delta\left(a^{\dagger} a+\frac{1}{2}\right)^{2}\right] \\
& \omega_{\mathrm{ch}}=\frac{|e| H}{m_{\mathrm{h}} c} ; \quad \delta=\frac{\left|\delta_{\mathrm{h}} E_{z}\right| \hbar^{4}}{l^{4} \hbar \omega_{\mathrm{ch}}}>0 .
\end{aligned}
$$

The RSOC Hamiltonian (8) in differential form can be transcribed

$$
\begin{gathered}
\hat{H}_{\mathrm{h}}^{\mathrm{SOC}}=\mathrm{i} \beta_{\mathrm{h}} E_{z}\left\{\hat{\sigma}_{x}\left[\hat{K}_{y}^{3}-\left(\hat{K}_{x}^{2} \hat{K}_{y}+\hat{K}_{y} \hat{K}_{x}^{2}+\hat{K}_{x} \hat{K}_{y} \hat{K}_{x}\right)\right]\right. \\
\left.+\hat{\sigma}_{y}\left[\hat{K}_{x}^{3}-\left(\hat{K}_{y}^{2} \hat{K}_{x}+\hat{K}_{x} \hat{K}_{y}^{2}+\hat{K}_{y} \hat{K}_{x} \hat{K}_{y}\right)\right]\right\}
\end{gathered}
$$

whereas in the second quantization representation it is

$$
\hat{H}_{\mathrm{h}}^{\mathrm{SOC}}=\mathrm{i} \beta 2 \sqrt{2} \hbar \omega_{\mathrm{ch}}\left|\begin{array}{cc}
0 & \left(a^{\dagger}\right)^{3} \\
-a^{3} & 0
\end{array}\right| ; \quad \beta=\frac{\beta_{\mathrm{h}} E_{z}}{l^{3} \hbar \omega_{\mathrm{ch}}} .
$$

The full Landau-Rashba Hamiltonian has the form

$$
\begin{aligned}
\hat{H}= & \hbar \omega_{\mathrm{ch}}\left\{\left[\left(a^{\dagger} a+\frac{1}{2}\right)+\delta\left(a^{\dagger} a+\frac{1}{2}\right)^{2}\right] \hat{I}\right. \\
& \left.+\mathrm{i} \beta 2 \sqrt{2}\left|\begin{array}{cc}
0 & \left(a^{\dagger}\right)^{3} \\
-a^{3} & 0
\end{array}\right|\right\} .
\end{aligned}
$$

Here the unit 2D matrix $I=\left|\begin{array}{cc}1 & 0 \\ 0 & 1\end{array}\right|$ was introduced.

The solution of the Schrödinger-Pauli-type Hamiltonian (20) was chosen in spinor form with column wavefunction

$$
\hat{H}\left|\begin{array}{l}
f_{1} \\
f_{2}
\end{array}\right|=E_{\mathrm{h}}\left|\begin{array}{l}
f_{1} \\
f_{2}
\end{array}\right| ; \quad E_{\mathrm{h}}=\hbar \omega_{\mathrm{ch}} \varepsilon_{\mathrm{h}}
$$

It is equivalent to write

$$
\begin{gathered}
{\left[\left(a^{\dagger} a+\frac{1}{2}\right)+\delta\left(a^{\dagger} a+\frac{1}{2}\right)^{2}\right] f_{1}+\mathrm{i} \beta 2 \sqrt{2}\left(a^{\dagger}\right)^{3} f_{2}=\varepsilon_{\mathrm{h}} f_{1}} \\
{\left[\left(a^{\dagger} a+\frac{1}{2}\right)+\delta\left(a^{\dagger} a+\frac{1}{2}\right)^{2}\right] f_{2}-\mathrm{i} \beta 2 \sqrt{2}(a)^{3} f_{1}=\varepsilon_{\mathrm{h}} f_{2}}
\end{gathered}
$$

The functions $f_{1}$ and $f_{2}$ are expressed through the Fock states

$$
\begin{gathered}
f_{1}=\sum_{n=0}^{\infty} c_{n}|n\rangle ; \quad f_{2}=\sum_{n=0}^{\infty} d_{n}|n\rangle ; \\
\sum_{n=0}^{\infty}\left|c_{n}\right|^{2}+\sum_{n=0}^{\infty}\left|d_{n}\right|^{2}=1 .
\end{gathered}
$$

Taking into account the equalities

$$
\begin{gathered}
a^{\dagger}|n\rangle=\sqrt{(n+1)(n+2)(n+3)}|n+3\rangle ; \\
a^{3}|n\rangle=\sqrt{n(n-1)(n-2)}|n-3\rangle ; \\
a^{\dagger} a|n\rangle=n|n\rangle,
\end{gathered}
$$

equations (21) will transform into

$$
\begin{aligned}
& \sum_{n=0}^{\infty} c_{n}\left[n+\frac{1}{2}+\delta\left(n+\frac{1}{2}\right)^{2}-\varepsilon_{\mathrm{h}}\right]|n\rangle \\
& \quad+\mathrm{i} \beta 2 \sqrt{2} \sum_{n=0}^{\infty} d_{n} \sqrt{(n+1)(n+2)(n+3)}|n+3\rangle=0 \\
& \sum_{n=0}^{\infty} d_{n}\left[n+\frac{1}{2}+\delta\left(n+\frac{1}{2}\right)^{2}-\varepsilon_{\mathrm{h}}\right]|n\rangle \\
& \quad-\mathrm{i} \beta 2 \sqrt{2} \sum_{n=0}^{\infty} c_{n} \sqrt{n(n-1)(n-2)}|n-3\rangle=0
\end{aligned}
$$

Multiplying these equations by $\langle m|$ and using the properties (24), we will obtain the linear algebraic equations for the coefficients $c_{m}$ and $d_{m}$. For the first three values of $m=0-2$ they are

$$
\begin{gathered}
c_{0}\left(\frac{1}{2}+\delta-\varepsilon_{\mathrm{h}}\right)=0 ; \quad c_{1}\left(\frac{3}{2}+9 \delta-\varepsilon_{\mathrm{h}}\right)=0 ; \\
c_{2}\left(\frac{5}{2}+25 \delta-\varepsilon_{\mathrm{h}}\right)=0 .
\end{gathered}
$$

For any other values of $m \geq 3$ we have obtained the general equations

$$
\begin{aligned}
& c_{m}\left(m+\frac{1}{2}+\delta(2 m+1)^{2}-\varepsilon_{\mathrm{h}}\right) \\
& \quad=-\mathrm{i} \beta 2 \sqrt{2} \sqrt{m(m-1)(m-2)} d_{m-3} ; \\
& d_{m-3}\left(m-\frac{5}{2}+\delta(2 m-5)^{2}-\varepsilon_{\mathrm{h}}\right) \\
& \quad=\mathrm{i} \beta 2 \sqrt{2} \sqrt{m(m-1)(m-2)} c_{m} ; \\
& \left|c_{m}\right|^{2}+\left|d_{m-3}\right|^{2}=1 .
\end{aligned}
$$

They lead to the dispersion equation

$$
\begin{gathered}
\left(m+\frac{1}{2}+\delta(2 m+1)^{2}-\varepsilon_{\mathrm{h}}\right)\left(m-\frac{5}{3}+\delta(2 m-5)^{2}-\varepsilon_{\mathrm{h}}\right) \\
=8 \beta^{2} m(m-1)(m-2) ; \quad m \geq 3 .
\end{gathered}
$$

Its solution $\varepsilon_{\mathrm{h}}$ depends simultaneously on two Landau level numbers $(m+1 / 2)$ and $(m-5 / 2)$ and will be denoted as $\varepsilon_{\mathrm{h}}(m-5 / 2 ; m+1 / 2)$. The exact solution of equation (28) is

$$
\begin{aligned}
& \varepsilon_{\mathrm{h}}^{ \pm}(m-5 / 2 ; m+1 / 2) \\
& =(m-1)+\frac{\delta}{2}\left[(2 m+1)^{2}+(2 m-5)^{2}\right] \\
& \quad \pm\left\{\left(\frac{3}{2}+\frac{\delta}{2}\left[(2 m+1)^{2}-(2 m-5)^{2}\right]\right)^{2}\right. \\
& \left.\quad+8 \beta^{2} m(m-1)(m-2)\right\}^{1 / 2}, \quad m \geq 3 .
\end{aligned}
$$

The Landau quantization task in the presence of the RSOC can be solved exactly. In the case of a conduction electron with a linear term $K_{ \pm}$in equation (5) this was proved earlier by Rashba [1, 5]. The case $K_{ \pm}^{2}$ was considered in [29]. Now we have proved the same in the case of holes with cubic term $K_{ \pm}^{3}$ in equation (18) and quartic term $k_{\|}^{4}$ in the Hamiltonian (13). 


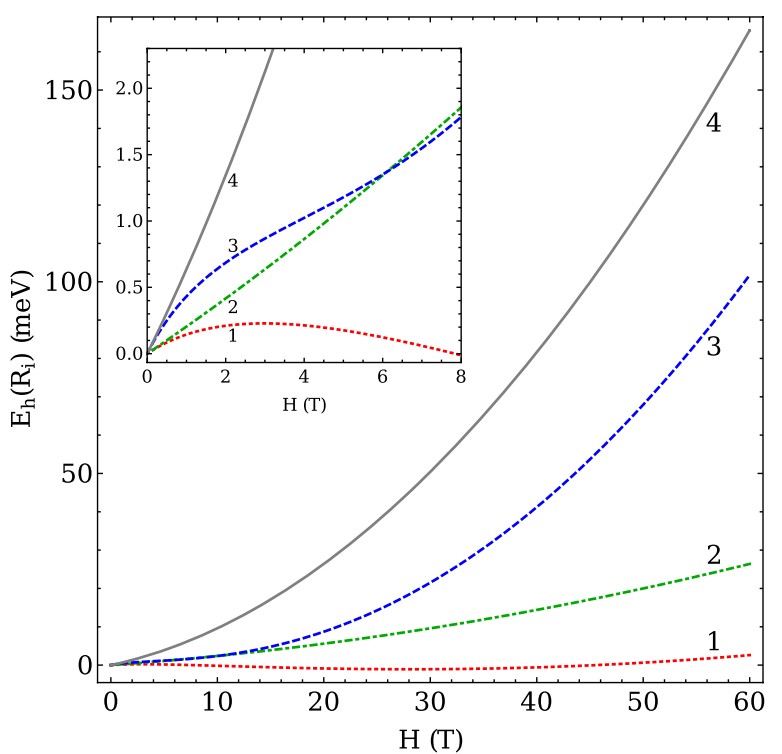

Figure 2. The dependences of the four lowest Landau levels $E_{\mathrm{h}}\left(R_{i}\right)$ with $i=1-4$ on the magnetic field strength measured in units of tesla at the parameter $E_{z}=10 \mathrm{kV} \mathrm{cm}{ }^{-1}$ and the parameter $\delta$ with $c=10$.

In the particular case $\beta=0$, the two solutions (29) become

$$
\begin{aligned}
& \varepsilon_{\mathrm{h}}^{+}\left(m+\frac{1}{2}\right)=m+\frac{1}{2}+\delta(2 m+1)^{2} \\
& \varepsilon_{\mathrm{h}}^{-}\left(m-\frac{5}{2}\right)=m-\frac{5}{2}+\delta(2 m-5)^{2} ; \quad m \geq 3 .
\end{aligned}
$$

The parameters $\beta$ and $\delta$ depend on the electric and magnetic field strengths $E_{z}=x \mathrm{kV} \mathrm{cm}{ }^{-1}$ and $H=y T$ in the forms $\beta=1.2 \times 10^{-2} x \sqrt{y}$ and $\delta=10^{-4} c x y$.

The four lowest Landau levels for a heavy hole with $m_{\mathrm{h}}=0.25 m_{0}$ being multiplied by $\hbar \omega_{\mathrm{ch}}=0.4 y \mathrm{meV}$ are

$$
\begin{array}{ll}
E_{\mathrm{h}}\left(R_{1}\right)=\hbar \omega_{\mathrm{ch}} \varepsilon_{\mathrm{h}}^{-}\left(\frac{1}{2}, \frac{7}{2}\right), & E_{\mathrm{h}}\left(R_{2}\right)=\hbar \omega_{\mathrm{ch}}\left(\frac{1}{2}+\delta\right), \\
E_{\mathrm{h}}\left(R_{3}\right)=\hbar \omega_{\mathrm{ch}} \varepsilon_{\mathrm{h}}^{-}\left(\frac{3}{2}, \frac{9}{2}\right), & E_{\mathrm{h}}\left(R_{4}\right)=\hbar \omega_{\mathrm{ch}}\left(\frac{3}{2}+9 \delta\right) .
\end{array}
$$

They are represented in figure 2 .

The state $E_{\mathrm{h}}\left(R_{1}\right)$ has a nonmonotonic dependence on the magnetic field strength $H$. The inset of figure 2 shows a positive maximal value of $0.23 \mathrm{meV}$ at $H=3 \mathrm{~T}$ accompanied by a slowly decreasing behaviour with a negative minimal value of $-1 \mathrm{meV}$ at $H=28 \mathrm{~T}$, followed by a monotonic slow increase

The danger of obtaining negative values and of penetrating deeply and unlimitedly inside the semiconductor energy gap revealed in the range $H=28 \mathrm{~T}$ was stopped and avoided due to the presence of the $\delta$ term in the Hamiltonian (13). The product $\delta \hbar \omega_{\mathrm{ch}}$ in expressions (31), where each factor is proportional to $H$, leads to the quadratic dependence $H^{2}$, which is observed in figure 2, especially looking at another three dependences $E_{\mathrm{h}}\left(R_{2}\right), E_{\mathrm{h}}\left(R_{3}\right)$ and $E_{\mathrm{h}}\left(R_{4}\right)$. They have a linear dependence on $H$ in the range of $H \leq(10-15) \mathrm{T}$, where the term $\delta=10^{-2} y<1$ is small in comparison with 1 and obtain a quadratic dependence at higher values of $H$.
The spinor parts of the four hole wavefunctions are

$$
\begin{gathered}
\left|\Psi_{\mathrm{h}}\left(R_{1}, q ; x, y\right)\right\rangle=\frac{\mathrm{e}^{\mathrm{i} q x}}{\sqrt{L_{x}}}\left|\begin{array}{c}
c_{3} \varphi_{3}(\eta) \\
d_{0} \varphi_{0}(\eta)
\end{array}\right| \\
\left|\Psi_{\mathrm{h}}\left(R_{2}, q ; x, y\right)\right\rangle=\frac{\mathrm{e}^{\mathrm{i} q x}}{\sqrt{L_{x}}}\left|\begin{array}{c}
\varphi_{0}(\eta) \\
0
\end{array}\right| ; \\
\left|\Psi_{\mathrm{h}}\left(R_{3}, q ; x, y\right)\right\rangle=\frac{\mathrm{e}^{\mathrm{i} q x}}{\sqrt{L_{x}}}\left|\begin{array}{c}
c_{4} \varphi_{4}(\eta) \\
d_{1} \varphi_{1}(\eta)
\end{array}\right| \\
\left|\Psi_{\mathrm{h}}\left(R_{4}, q ; x, y\right)\right\rangle=\frac{\mathrm{e}^{\mathrm{i} q x}}{\sqrt{L_{x}}}\left|\begin{array}{c}
\varphi_{1}(\eta) \\
0
\end{array}\right| \\
\eta=\frac{y}{l}+q l .
\end{gathered}
$$

The valence electrons in the same conditions are described by an equivalent set of spinor wavefunctions

$$
\left|\Psi_{\mathrm{v}}\left(R_{j}, q ; x, y\right)\right\rangle=\mathrm{i} \hat{\sigma}_{y}\left|\Psi_{\mathrm{h}}\left(R_{j},-q ; x, y\right)\right\rangle^{*},
$$

so that their spin projections and wavevectors have opposite directions in comparison with those of the holes. To obtain the conduction and valence electron Bloch wavefunctions the spinor wavefunctions (33) must be multiplied by the corresponding periodic parts. They are needed for the calculations in the next sections. For valence electrons the periodic parts are chosen the same as in the absence of the RSOC, because the latter cannot change the basic electron band structure of the semiconductor.

Now for completeness we will recall the main results obtained by Rashba in the case of the electron conduction band. They are needed to obtain a full description of the $2 \mathrm{D}$ $\mathrm{e}-\mathrm{h}$ pair and of a $2 \mathrm{D}$ magnetoexciton in the condition of a Landau quantization under the influence of the RSOC.

The lowest Landau level of the conduction electron in the presence of the RSOC was obtained in [1]:

$$
\begin{gathered}
\left|\Psi_{\mathrm{e}}\left(R_{1}, p ; x_{\mathrm{e}}, y_{\mathrm{e}}\right)\right\rangle=\frac{\mathrm{e}^{\mathrm{i} p x_{\mathrm{e}}}}{\sqrt{L_{x}}}\left|\begin{array}{l}
a_{0} \varphi_{0}\left(y_{\mathrm{e}}\right) \\
b_{1} \varphi_{1}\left(y_{\mathrm{e}}\right)
\end{array}\right| \\
\varepsilon_{\mathrm{e} R_{1}}=1-\sqrt{\frac{1}{4}+2 \alpha^{2}} ; \quad\left|a_{0}\right|^{2}+\left|b_{1}\right|^{2}=1 \\
\left|a_{0}\right|^{2}=\frac{1}{1+\frac{2 \alpha^{2}}{\left[\frac{1}{2}+\sqrt{\frac{1}{4}+2 \alpha^{2}}\right]^{2}}} ; \\
\left|b_{1}\right|^{2}=\frac{2 \alpha^{2}\left|a_{0}\right|^{2}}{\left[\frac{1}{2}+\sqrt{\frac{1}{4}+2 \alpha^{2}}\right]^{2}}
\end{gathered}
$$

The next electron level situated higher on the energy scale is characterized by the pure spin oriented state

$$
\left|\Psi_{\mathrm{e}}\left(R_{2}, p ; x_{\mathrm{e}}, y_{\mathrm{e}}\right)\right\rangle=\frac{\mathrm{e}^{\mathrm{i} p x_{\mathrm{e}}}}{\sqrt{L_{x}}}\left|\begin{array}{c}
0 \\
\varphi_{0}\left(y_{\mathrm{e}}\right)
\end{array}\right| ; \quad \varepsilon_{\mathrm{e} R_{2}}=\frac{1}{2} .
$$

The two lowest Landau levels (LLLs) for the conduction electron are characterized by the values $m_{\mathrm{e}}=0.067 m_{0}$, $\hbar \omega_{\mathrm{ce}}=1.49 \mathrm{meV} \cdot y$ and the parameter $\alpha=8 \times 10^{-3} x / \sqrt{y}$. 
They are denoted as

$$
\begin{gathered}
E_{\mathrm{e}}\left(R_{1}\right)=\hbar \omega_{\text {ce }}\left(1-\sqrt{\frac{1}{4}+2 \alpha^{2}}\right) ; \\
E_{\mathrm{e}}\left(R_{2}\right)=\hbar \omega_{\text {ce }} \frac{1}{2} .
\end{gathered}
$$

The lowest Landau energy level for the electron $E_{\mathrm{e}}\left(R_{1}\right)$ has a nonmonotonic anomalous dependence on the magnetic field strength near the point $H=0 \mathrm{~T}$. It is due to the singular dependence of the RSOC parameter $\alpha^{2}=6.4 \times 10^{-5} x^{2} / y$, which is compensated in the total energy level expression by the factor $\hbar \omega_{\mathrm{ce}}$ of the cyclotron energy, where $\hbar \omega_{\mathrm{ce}}=$ $1.49 y \mathrm{meV}$. The second electron Landau energy level has a simple linear dependence on $H$.

The combinations of two LLLs for a conduction electron with four LLLs for an $\mathrm{HH}$ give rise to eight possible band-to-band transitions. They will be denoted as $F_{n}\left(e R_{i} ; h R_{j}\right)$, where $n=1-8, i=1,2$ and $j=1-4$. The energies of the corresponding band-to-band transitions $E_{\mathrm{cv}}\left(F_{n}\right)$ without the semiconductor energy gap $E_{\mathrm{g}}$ are equal to

$$
\begin{aligned}
& E_{\mathrm{cv}}\left(F_{1}\right)-E_{\mathrm{g}}=E_{\mathrm{e}}\left(R_{1}\right)+E_{\mathrm{h}}\left(R_{1}\right), \\
& E_{\mathrm{cv}}\left(F_{2}\right)-E_{\mathrm{g}}=E_{\mathrm{e}}\left(R_{2}\right)+E_{\mathrm{h}}\left(R_{1}\right), \\
& E_{\mathrm{cv}}\left(F_{3}\right)-E_{\mathrm{g}}=E_{\mathrm{e}}\left(R_{1}\right)+E_{\mathrm{h}}\left(R_{2}\right), \\
& E_{\mathrm{cv}}\left(F_{4}\right)-E_{\mathrm{g}}=E_{\mathrm{e}}\left(R_{2}\right)+E_{\mathrm{h}}\left(R_{2}\right), \\
& E_{\mathrm{cv}}\left(F_{5}\right)-E_{\mathrm{g}}=E_{\mathrm{e}}\left(R_{1}\right)+E_{\mathrm{h}}\left(R_{3}\right), \\
& E_{\mathrm{cv}}\left(F_{6}\right)-E_{\mathrm{g}}=E_{\mathrm{e}}\left(R_{2}\right)+E_{\mathrm{h}}\left(R_{3}\right), \\
& E_{\mathrm{cv}}\left(F_{7}\right)-E_{\mathrm{g}}=E_{\mathrm{e}}\left(R_{1}\right)+E_{\mathrm{h}}\left(R_{4}\right), \\
& E_{\mathrm{cv}}\left(F_{8}\right)-E_{\mathrm{g}}=E_{\mathrm{e}}\left(R_{2}\right)+E_{\mathrm{h}}\left(R_{4}\right) .
\end{aligned}
$$

Their dependences on the magnetic field strength at the parameters $E_{z}=10 \mathrm{kV} \mathrm{cm}^{-1}$ and $c=10$ are shown in figure 3. As one can see the LLLs for electrons and HHs do not penetrate deeply inside the semiconductor energy gap $E_{\mathrm{g}}$, if the parameter $\delta$ is not too small. The introduction of the parameter $\delta$ allows us to avoid the unphysical shrinkage of the energy gap between the valence and conduction bands.

In figure 3(a) the energies $E_{\mathrm{cv}}\left(F_{n}\right)-E_{\mathrm{g}}$ of the band-to-band quantum transitions in a large range of magnetic field strength are represented. The hole level $h R_{1}$ gives rise to quantum transitions $F_{1}$ and $F_{2}$ to the electron states $e R_{1}$ and $e R_{2}$. The hole level $h R_{2}$ gives rise to the quantum transitions $F_{3}$ and $F_{4}$ to the same electron states $e R_{1}$ and $e R_{2}$. Another two hole levels $h R_{3}$ and $h R_{4}$ give rise to two groups of quantum transitions $F_{5}, F_{6}$ and $F_{7}, F_{8}$ correspondingly. The dependences $E_{\mathrm{cv}}\left(F_{n}\right)-E_{\mathrm{g}}$ of these eight quantum transitions are represented in figure 3(b) in the range of $H<0.5 \mathrm{~T}$. They differ essentially from figure 3(a) and are determined not only by the hole Landau levels but also by the conduction electron Landau levels.

The obtained results concerning the electron and hole LLLs as well as the energies $E_{\mathrm{cv}}\left(F_{n}\right)$ of the band-to-band quantum transitions will be used to determine the lowest levels of the magnetoexciton states. The Coulomb e-h interaction in the frame of these magnetoexciton states will be studied in section 3 .
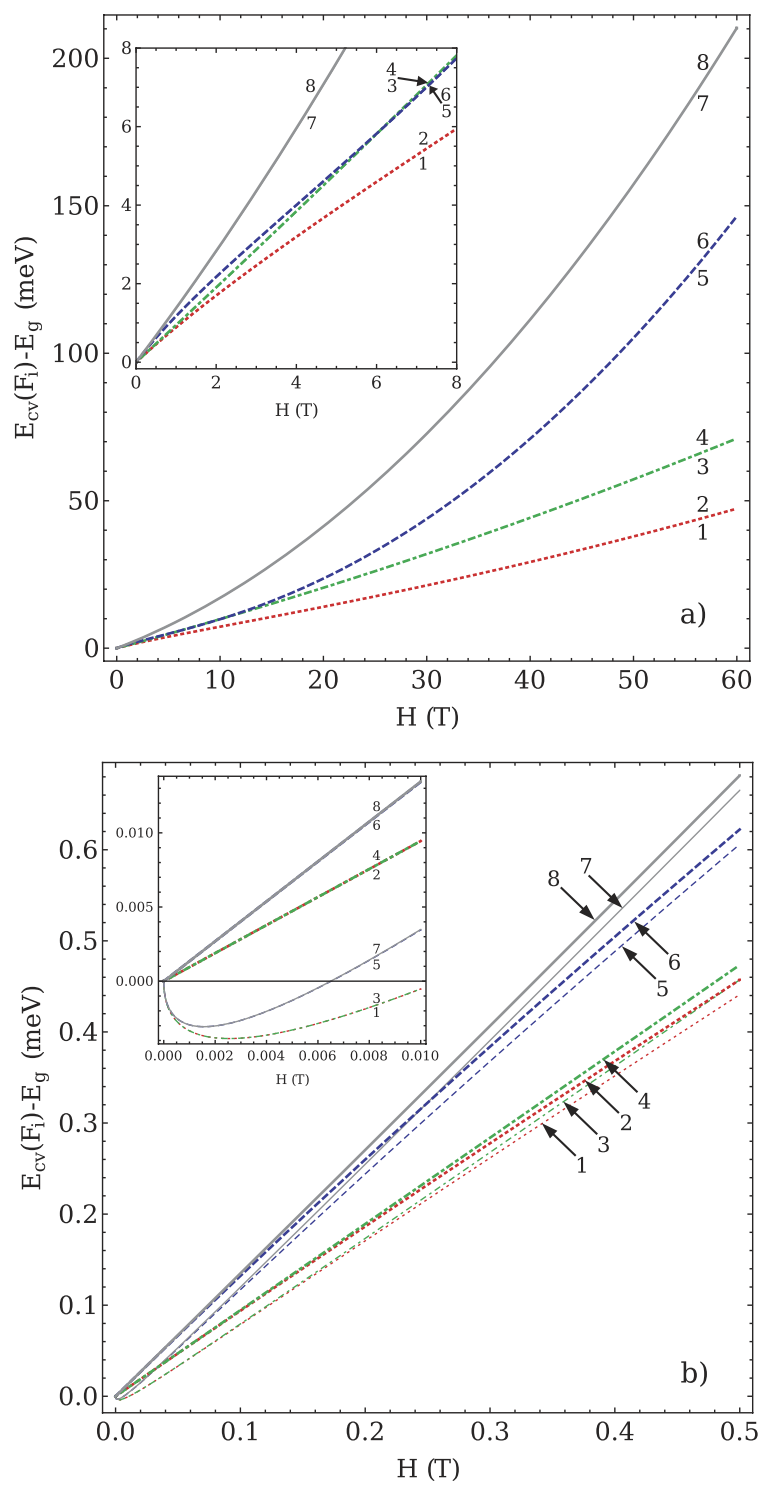

Figure 3. The dependences on the magnetic field strengths of the band-to-band quantum transition energies $E_{\mathrm{cv}}\left(F_{n}\right)$ for eight combinations of two LLLs of a conduction electron with four LLLs for an $\mathrm{HH}$ with the parameters $E_{z}=10 \mathrm{kV} \mathrm{cm}^{-1}$ and $c=10$. (a) A large interval of magnetic field strength; (b) a small interval of the values of $H$.

\section{The Coulomb electron-hole interaction and the energy spectrum of the two-dimensional magnetoexcitons}

The $\mathrm{e}-\mathrm{h}$ Coulomb interaction is calculated below taking into account the influence of the RSOC in the frame of the conduction and valence bands. The corresponding Bloch wavefunctions including their periodic parts are

$$
\begin{aligned}
& \left|\Psi_{\mathrm{c}}\left(R_{1}, p ; x, y\right)\right\rangle=\frac{\mathrm{e}^{\mathrm{i} p x}}{\sqrt{L_{x}}} U_{c, s, p}(\vec{r})\left|\begin{array}{l}
a_{0} \varphi_{0}\left(\eta_{\mathrm{c}}\right) \\
b_{1} \varphi_{1}\left(\eta_{\mathrm{c}}\right)
\end{array}\right| ; \\
& \left|\Psi_{\mathrm{c}}\left(R_{2}, p ; x, y\right)\right\rangle=\frac{\mathrm{e}^{\mathrm{i} p x}}{\sqrt{L_{x}}} U_{c, s, p}(\vec{r})\left|\begin{array}{c}
0 \\
\varphi_{0}\left(\eta_{\mathrm{c}}\right)
\end{array}\right| ; \\
& \eta_{\mathrm{c}}=\frac{y}{l}-p l ;
\end{aligned}
$$




$$
\begin{aligned}
& \left|\Psi_{\mathrm{v}}\left(R_{1}, q ; x, y\right)\right\rangle=\frac{\mathrm{e}^{\mathrm{i} q x}}{\sqrt{L_{x}}} \frac{1}{\sqrt{2}}\left(U_{v, P, X, q}(\vec{r})\right. \\
& \left.+\mathrm{i} U_{v, P, Y, q}(\vec{r})\right)\left|\begin{array}{c}
d_{0} \varphi_{0}\left(\eta_{\mathrm{v}}\right) \\
-c_{3} \varphi_{3}\left(\eta_{\mathrm{v}}\right)
\end{array}\right| ; \\
& \left|\Psi_{\mathrm{v}}\left(R_{2}, q ; x, y\right)\right\rangle=\frac{\mathrm{e}^{\mathrm{i} q x}}{\sqrt{L_{x}}} \frac{1}{\sqrt{2}}\left(U_{v, P, X, q}(\vec{r})\right. \\
& \left.-\mathrm{i} U_{v, P, Y, q}(\vec{r})\right)\left|\begin{array}{c}
0 \\
-\varphi_{0}\left(\eta_{\mathrm{v}}\right)
\end{array}\right| \text {; } \\
& \left|\Psi_{\mathrm{v}}\left(R_{3}, q ; x, y\right)\right\rangle=\frac{\mathrm{e}^{\mathrm{i} q x}}{\sqrt{L_{x}}} \frac{1}{\sqrt{2}}\left(U_{v, P, X, q}(\vec{r})\right. \\
& \left.+\mathrm{i} U_{v, P, Y, q}(\vec{r})\right)\left|\begin{array}{c}
d_{1} \varphi_{1}\left(\eta_{\mathrm{v}}\right) \\
-c_{4} \varphi_{4}\left(\eta_{\mathrm{v}}\right)
\end{array}\right| ; \\
& \left|\Psi_{\mathrm{v}}\left(R_{4}, q ; x, y\right)\right\rangle=\frac{\mathrm{e}^{\mathrm{i} q x}}{\sqrt{L_{x}}} \frac{1}{\sqrt{2}}\left(U_{v, P, X, q}(\vec{r})\right. \\
& \left.-\mathrm{i} U_{v, P, Y, q}(\vec{r})\right)\left|\begin{array}{c}
0 \\
-\varphi_{1}\left(\eta_{\mathrm{v}}\right)
\end{array}\right| \text {; } \\
& \eta_{\mathrm{v}}=\frac{y}{l}-q l .
\end{aligned}
$$

Here $U_{c, s, p}(\vec{r})$ is the s-type conduction band periodic part, whereas $\frac{1}{\sqrt{2}}\left(U_{v, P, X, q}(\vec{r}) \pm \mathrm{i} U_{v, P, Y, q}(\vec{r})\right)$ are the p-type valence band periodic parts.

The orthogonality between the conduction and valence electron Bloch wavefunctions is attained due to their orthogonal periodic parts, whereas the orthogonality of the wavefunctions belonging to the same bands and having the same periodic parts is reached due to different numbers of Landau quantization wavefunctions $\varphi_{\mathrm{c}, n}(y, p)$ and $\varphi_{\mathrm{v}, m}(y, p)$. The conduction and valence electrons have the same electric charge $-|e|$ and their dimensionless variables have the same structure $\frac{y}{l}-p l$ and $\frac{y}{l}-q l$. The last variable is $\frac{y}{l}+q l$ in the case of the hole wavefunction $\varphi_{\mathrm{h}, n}(y, q)$ due to the positive value of the hole charge $|e|$.

The Hamiltonian of the Coulomb electron-electron interaction can be calculated in the same way as was demonstrated in the paper [30]. Differently from it we have dealt with spinor-type wavefunctions for electrons and holes with a column representation (38). The creation and annihilation operators for conduction and valence electrons are denoted as $a_{c, R_{i}, p}^{\dagger}, a_{c, R_{i}, p}, a_{v, R_{j}, q}^{\dagger}, a_{v, R_{j}, q}$. The Hamiltonian of their Coulomb interaction has the form

$$
\begin{aligned}
& \sum_{p, q, s} F_{\mathrm{c}-\mathrm{v}}\left(c, R_{i}, p ; v, R_{j}, q ; c, R_{i}, p-s ; v, R_{j}, q+s\right) \\
& \quad \times a_{c, R_{i}, p}^{\dagger} a_{v, R_{j}, q}^{\dagger} a_{v, R_{j}, q+s} a_{c, R_{i}, p-s .}
\end{aligned}
$$

The wavefunction of a magnetoexciton state created by the electron on the Landau quantization level $e R_{i}$ and by the hole on the hole state $h R_{j}$ has the form

$$
\begin{gathered}
\Psi_{\mathrm{ex}}\left(\vec{k}, f_{n}\right)=\frac{1}{\sqrt{N}} \sum_{t} \mathrm{e}^{-\mathrm{i} k_{y} t l^{2}} a_{R_{i}, \frac{k_{x}}{2}+t}^{\dagger} b_{R_{j}, \frac{k_{x}}{2}-t}^{\dagger}|0\rangle ; \\
n=1-8 ; \quad i=1,2 ; \quad j=1-4 .
\end{gathered}
$$

Here the hole operators $b_{R_{j}, q}^{\dagger}=a_{v, R_{j},-q}$ and $b_{R_{j}, q}=a_{v, R_{j},-q}^{\dagger}$ were introduced. Using the hole operators the Coulomb repulsive $F_{\mathrm{c}-\mathrm{v}}$ interaction is transformed into the attractive Coulomb e-h interaction $F_{\mathrm{e}-\mathrm{h}}$, the average value of which is

$$
\begin{gathered}
\left\langle\Psi_{\mathrm{ex}}\left(\vec{k}, f_{n}\right)\left|H_{\mathrm{Coul}}^{\mathrm{e}-\mathrm{h}}\right| \Psi_{\mathrm{ex}}\left(\vec{k}, f_{n}\right)\right\rangle=-\frac{1}{N} \sum_{p, s} \mathrm{e}^{\mathrm{i} k_{y} s l^{2}} \\
\times F_{\mathrm{e}-\mathrm{h}}\left(e, R_{i}, p ; h, R_{j}, k_{x}-p ; e, R_{i}, p-s ;\right. \\
\left.h, R_{j}, k_{x}+s-p\right)=-I_{\mathrm{ex}}\left(e, R_{i} ; h, R_{j} ; \vec{k}\right) .
\end{gathered}
$$

Taking into account the spinor-type conduction and valence electron wavefunction as well as the results of [30], we obtain

$$
\begin{aligned}
& I_{\mathrm{ex}}\left(F_{1}, \vec{k}\right)=I_{\mathrm{ex}}\left(e, R_{1} ; h, R_{1} ; \vec{k}\right) \\
& =\left|a_{0}\right|^{2}\left|d_{0}\right|^{2} I_{\mathrm{ex}}^{(0,0)}(\vec{k})+\left|a_{0}\right|^{2}\left|c_{3}\right|^{2} I_{\mathrm{ex}}^{(0,3)}(\vec{k}) \\
& \quad+\left|d_{0}\right|^{2}\left|b_{1}\right|^{2} I_{\mathrm{ex}}^{(0,1)}(\vec{k})+\left|b_{1}\right|^{2}\left|c_{3}\right|^{2} I_{\mathrm{ex}}^{(1,3)}(\vec{k}) ; \\
& I_{\mathrm{ex}}\left(F_{2}, \vec{k}\right)=I_{\mathrm{ex}}\left(e, R_{2} ; h, R_{1} ; \vec{k}\right) \\
& =\left|d_{0}\right|^{2} I_{\mathrm{ex}}^{(0,0)}(\vec{k})+\left|c_{3}\right|^{2} I_{\mathrm{ex}}^{(0,3)}(\vec{k}) ; \\
& I_{\mathrm{ex}}\left(F_{3}, \vec{k}\right)=I_{\mathrm{ex}}\left(e, R_{1} ; h, R_{2} ; \vec{k}\right) \\
& =\left|a_{0}\right|^{2} I_{\mathrm{ex}}^{(0,0)}(\vec{k})+\left|b_{1}\right|^{2} I_{\mathrm{ex}}^{(0,1)}(\vec{k}) ; \\
& I_{\mathrm{ex}}\left(F_{4}, \vec{k}\right)=I_{\mathrm{ex}}\left(e, R_{2} ; h, R_{2} ; \vec{k}\right)=I_{\mathrm{ex}}^{(0,0)}(\vec{k}) ; \\
& I_{\mathrm{ex}}\left(F_{5}, \vec{k}\right)=I_{\mathrm{ex}}\left(e, R_{1} ; h, R_{3} ; \vec{k}\right) \\
& =\left|a_{0}\right|^{2}\left|c_{4}\right|^{2} I_{\mathrm{ex}}^{(0,4)}(\vec{k})+\left|a_{0}\right|^{2}\left|d_{1}\right|^{2} I_{\mathrm{ex}}^{(0,1)}(\vec{k}) \\
& \quad+\left|b_{1}\right|^{2}\left|c_{4}\right|^{2} I_{\mathrm{ex}}^{(1,4)}(\vec{k})+\left|b_{1}\right|^{2}\left|d_{1}\right|^{2} I_{\mathrm{ex}}^{(1,1)}(\vec{k}) ; \\
& I_{\mathrm{ex}}\left(F_{6}, \vec{k}\right)=I_{\mathrm{ex}}\left(e, R_{2} ; h, R_{3} ; \vec{k}\right) \\
& =\left|c_{4}\right|^{2} I_{\mathrm{ex}}^{(0,4)}(\vec{k})+\left|d_{1}\right|^{2} I_{\mathrm{ex}}^{(0,1)}(\vec{k}) ; \\
& I_{\mathrm{ex}}\left(F_{7}, \vec{k}\right)=I_{\mathrm{ex}}\left(e, R_{1} ; h, R_{4} ; \vec{k}\right) \\
& \quad=\left|a_{0}\right|^{2} I_{\mathrm{ex}}^{(0,1)}(\vec{k})+\left|b_{1}\right|^{2} I_{\mathrm{ex}}^{(1,1)}(\vec{k}) ; \\
& I_{\mathrm{ex}}\left(F_{8}, \vec{k}\right)=I_{\mathrm{ex}}\left(e, R_{2} ; h, R_{4} ; \vec{k}\right)=I_{\mathrm{ex}}^{(0,1)}(\vec{k}) .
\end{aligned}
$$

The creation energies of the eight magnetoexciton states are

$$
E_{\mathrm{ex}}\left(F_{n}, \vec{k}\right)=E_{\mathrm{cv}}\left(F_{n}\right)-I_{\mathrm{ex}}\left(F_{n}, \vec{k}\right) .
$$

Their dependences on the magnetic field strength $H$ can be demonstrated only in the range $H \geq 7 \mathrm{~T}$, because the magnetoexcitons exist only in the range of high perpendicular magnetic field.

One can remember that in the GaAs-type crystal the electron cyclotron energy $\hbar \omega_{\text {ce }}$ becomes comparable to and larger than the 2D Wannier-Mott exciton binding energy and at the same time the magnetic length $l$ becomes smaller than the exciton Bohr radius just at the values $H \geq 7 \mathrm{~T}$. Of most interest are the four lowest exciton energy levels at the point $k=0$ where the optical quantum transitions take place. These levels are $E_{\mathrm{ex}}\left(F_{1}, 0\right), E_{\mathrm{ex}}\left(F_{2}, 0\right), E_{\mathrm{ex}}\left(F_{3}, 0\right)$ and $E_{\mathrm{ex}}\left(F_{6}, 0\right)$. Their positions on the energy scale and dependences on the magnetic field strength are represented in figure 4 in the actual range of magnetic field.

The creation energies of the lowest magnetoexciton levels $F_{1}$ and $F_{2}$ at the point $k=0$ depending on the magnetic field strength $H>7 \mathrm{~T}$ have a general increasing behaviour with small minima in the vicinity of $H=20 \mathrm{~T}$. 

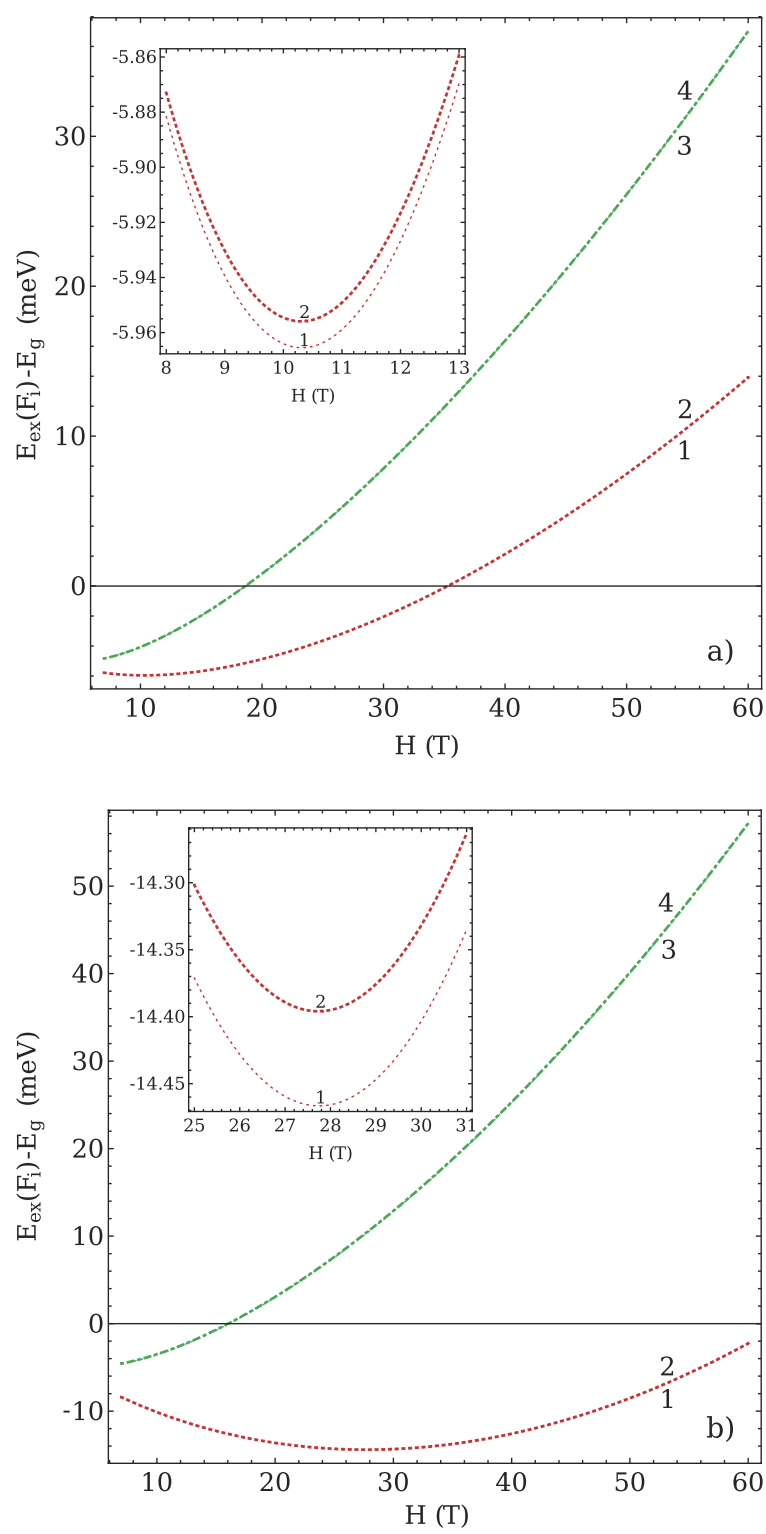

Figure 4. The dependences of the creation energies of the four lowest magnetoexciton levels taken at the point $k=0$ on the magnetic field strength $H \geq 7 \mathrm{~T}$ at the electric field strength $E_{z}=10 \mathrm{kV} \mathrm{cm}^{-1}$ and the parameter $\delta$ with $c=10$.

The dependences of $E_{\mathrm{cv}}\left(F_{n}\right)$ on the magnetic field strength $H<7 \mathrm{~T}$ represented also in figure 3 present interest in the case of the 2D Wannier-Mott exciton, when the Coulomb e-h interaction is more important than the Landau quantization effects.

The probabilities of the quantum transitions in the lowest exciton states as well as in the case of band-to-band quantum transitions will be discussed in section 4 .

\section{Band-to-band quantum transitions, optical orientation, exciton alignment, and spin polarization in the presence of the Rashba spin-orbit coupling}

To deduce the selection rules and the probabilities of the quantum transitions from the ground state of the crystal to the magnetoexciton states as well as in the case of band-to-band quantum transitions one needs to know the Hamiltonian of the electron-radiation interaction describing the band-to-band transitions in the presence of the RSOC. Following the paper [31], using the electron creation and annihilation operators $a_{c, R_{i}, p}^{\dagger}, a_{c, R_{i}, p}, a_{v, R_{j}, q}^{\dagger}$ and $a_{v, R_{j}, q}$ introduced above, as well as the photon creation and annihilation operators $\left(C_{\vec{k}, \pm}\right)^{\dagger}, C_{\vec{k}, \pm}$ and conserving only the resonance terms of the Hamiltonian, one can write

$$
\begin{aligned}
H_{\mathrm{e}-\mathrm{rad}} & =\left(-\frac{e}{m_{0}}\right) \sum_{\vec{k}\left(k_{x}, k_{y}, k_{z}\right)} \sqrt{\frac{2 \pi \hbar}{V \omega_{k}}} \\
& \times \sum_{i=1,2} \sum_{j=1,2} \sum_{g, q}\left\{\left[C_{\vec{k},-}\left(\vec{\sigma}_{\vec{k}}^{+} \cdot \overrightarrow{\mathcal{P}}\left(c, R_{i}, g ; v, R_{j}, q ; \vec{k}\right)\right)\right.\right. \\
& \left.+C_{\vec{k},+}\left(\vec{\sigma}_{\vec{k}}^{-} \cdot \overrightarrow{\mathcal{P}}\left(c, R_{i}, g ; v, R_{j}, q ; \vec{k}\right)\right)\right] a_{c, R_{i}, g}^{\dagger} a_{v, R_{j}, q} \\
& +\left[\left(C_{\vec{k},+}\right)^{\dagger}\left(\vec{\sigma}_{\vec{k}}^{+} \cdot \overrightarrow{\mathcal{P}}\left(v, R_{j}, q ; c, R_{i}, g ;-\vec{k}\right)\right)\right. \\
& \left.\left.+\left(C_{\vec{k},-}\right)^{\dagger}\left(\vec{\sigma}_{\vec{k}}^{-} \cdot \overrightarrow{\mathcal{P}}\left(v, R_{j}, q ; c, R_{i}, g ;-\vec{k}\right)\right)\right] a_{v, R_{j}, q}^{\dagger} a_{c, R_{i}, g}\right\}
\end{aligned}
$$

One can remember that the optical orientation effects are related to the absorption of the circularly polarized radiation. In this case the light is characterized by the photon creation and annihilation operators $\left(C_{\vec{k}, \pm}\right)^{\dagger}$ and $C_{\vec{k}, \pm}$ and circular polarization vectors $\vec{\sigma}_{\vec{k}}^{ \pm}$determined as

$$
C_{\vec{k}, \pm}=\frac{1}{\sqrt{2}}\left(C_{\vec{k}, 1} \pm \mathrm{i} C_{\vec{k}, 2}\right) ; \quad \vec{\sigma}_{\vec{k}}^{ \pm}=\frac{1}{\sqrt{2}}\left(\vec{e}_{\vec{k}, 1} \pm \mathrm{i} \vec{e}_{\vec{k}, 2}\right) .
$$

Here $\vec{e}_{\vec{k}, 1}$ and $\vec{e}_{\vec{k}, 2}$ are unit vectors of linear polarization perpendicular to the wavevector $\vec{k}$.

The circularly polarized light propagates without confinement in the whole 3D space with volume $V$ and its direction is arbitrarily oriented as regards the $2 \mathrm{D}$ layer. The $\mathrm{e}-\mathrm{h}$ pairs and magnetoexcitons are lying on the surface of the layer. The heavy holes are created in the p-type valence band and are characterized by the orbital momentum projections $M= \pm 1$ in the direction of the external magnetic field perpendicular to the layer surface. These states are described introducing the circular polarization vectors $\vec{\sigma}_{ \pm 1}$ for the heavy holes and for the magnetoexcitons as follows:

$$
\vec{\sigma}_{ \pm 1}=\frac{1}{\sqrt{2}}\left(\vec{a}_{1} \pm \mathrm{i} \vec{a}_{2}\right)
$$

where $\vec{a}_{1}$ and $\vec{a}_{2}$ are the unit vectors lying in the plane of the layer. It is not surprising that the matrix elements of the light-matter interaction Hamiltonian happened to be expressed through the scalar products of the circular polarization vectors of the light and matter in the form $\left(\vec{\sigma}_{\vec{k}}^{ \pm *}\right.$. $\vec{\sigma}_{ \pm 1}$ ). Looking at the valence electron wavefunctions (38) one can observe that the states $v R_{1}$ and $v R_{3}$ are characterized by the projection $M_{\mathrm{v}}=+1$, whereas the states $v R_{2}$ and $v R_{4}$ are characterized by the quantum number $M_{\mathrm{v}}=-1$.

Taking into account that the hole in the valence band has the projection $M$ with the opposite sign in comparison with the valence electron we can determine the quantum 
number $M$ for the magnetoexciton states $F_{1}, F_{2}, F_{5}$ and $F_{6}$ as equal to -1 , whereas the states $F_{3}, F_{4}, F_{7}$ and $F_{8}$ are characterized by the quantum number $M=+1$. Other quantum numbers characterizing the magnetoexciton states are the $2 \mathrm{D}$ wavevector $\vec{k}$ and the numbers of Landau levels taking part in the formation of the RSOC states $e R_{i}$ and $h R_{j}$. All these quantum numbers determine the matrix elements of the Hamiltonian of the light-matter interaction (44), the probabilities of the quantum transitions as well as the effects of spin polarization and of magnetoexciton alignment.

The calculations of the matrix elements in (44) were made using the electron wavefunctions (38). The matrix elements which determine the quantum transitions $F_{n}$ are listed below. They can be represented in the form

$$
\begin{aligned}
\left(\vec{\sigma}_{\vec{k}}\right. & \left.\cdot \overrightarrow{\mathcal{P}}\left(v, R_{j}, q ; c, R_{i}, g ;-\vec{k}\right)\right)=\delta_{\mathrm{kr}}\left(q, g-k_{x}\right) \\
& \times P_{\mathrm{vc}}(g, k) \mathrm{e}^{-\mathrm{i} k_{y} g l^{2}} S\left(F_{n} ; v, R_{j} ; c, R_{i} ; \vec{k}\right),
\end{aligned}
$$

where the factors $S\left(F_{n} ; v, R_{j} ; c, R_{i} ; \vec{k}\right)$ are

$$
\begin{aligned}
& S\left(F_{1} ; v, R_{1} ; c, R_{1} ; \vec{k}\right)=\left(\vec{\sigma}_{k} \cdot \vec{\sigma}_{-1}\right) \\
& \quad \times\left[d_{0} a_{0} \Phi\left(v, 0,-k_{x} ; c, 0,0 ;-k_{y}\right)\right. \\
& \left.\quad-c_{3} b_{1} \Phi\left(v, 3,-k_{x} ; c, 1,0 ;-k_{y}\right)\right], \\
& S\left(F_{2} ; v, R_{1} ; c, R_{2} ; \vec{k}\right)=\left(\vec{\sigma}_{k} \cdot \vec{\sigma}_{-1}\right) \\
& \quad \times\left[-c_{3} \Phi\left(v, 3,-k_{x} ; c, 0,0 ;-k_{y}\right)\right], \\
& S\left(F_{3} ; v, R_{2} ; c, R_{1} ; \vec{k}\right)=\left(\vec{\sigma}_{k} \cdot \vec{\sigma}_{+1}\right) \\
& \quad \times\left[-b_{1} \Phi\left(v, 0,-k_{x} ; c, 1,0 ;-k_{y}\right)\right], \\
& S\left(F_{4} ; v, R_{2} ; c, R_{2} ; \vec{k}\right)=\left(\vec{\sigma}_{k} \cdot \vec{\sigma}_{+1}\right) \\
& \quad \times\left[-\Phi\left(v, 0,-k_{x} ; c, 0,0 ;-k_{y}\right)\right], \\
& S\left(F_{5} ; v, R_{3} ; c, R_{1} ; \vec{k}\right)=\left(\vec{\sigma}_{k} \cdot \vec{\sigma}_{-1}\right) \\
& \quad \times\left[d_{1} a_{0} \Phi\left(v, 1,-k_{x} ; c, 0,0 ;-k_{y}\right)\right. \\
& \left.\quad-c_{4} b_{1} \Phi\left(v, 4,-k_{x} ; c, 1,0 ;-k_{y}\right)\right], \\
& S\left(F_{6} ; v, R_{3} ; c, R_{2} ; \vec{k}\right)=\left(\vec{\sigma}_{k} \cdot \vec{\sigma}_{-1}\right) \\
& \quad \times\left[-c_{4} \Phi\left(v, 4,-k_{x} ; c, 0,0 ;-k_{y}\right)\right], \\
& S\left(F_{7} ; v, R_{4} ; c, R_{1} ; \vec{k}\right)=\left(\vec{\sigma}_{k} \cdot \vec{\sigma}_{+1}\right) \\
& \quad \times\left[-b_{1} \Phi\left(v, 1,-k_{x} ; c, 1,0 ;-k_{y}\right)\right], \\
& S\left(F_{8} ; v, R_{4} ; c, R_{2} ; \vec{k}\right)=\left(\vec{\sigma}_{k} \cdot \vec{\sigma}_{+1}\right) \\
& \quad \times\left[-\Phi\left(v, 1,-k_{x} ; c, 0,0 ;-k_{y}\right)\right] .
\end{aligned}
$$

Here $P_{\mathrm{vc}}$ is the matrix element of the band-to-band optical transition. In our case the transitions take place between the s-type conduction band and p-type valence band, they are of the allowed type following the Elliott classification and $P_{\mathrm{vc}}$ can be considered as a constant which does not depend on the wave numbers $Q_{y}$ and $g$ [31, 32]. In the expressions (48) there are also other factors, which play the roles of normalization and orthogonalization conditions with some corrections depending on the projections $k_{x}$ and $k_{y}$ of the light wavevector $\vec{k}$ on the layer plane. These integrals are

$$
\begin{aligned}
& \Phi\left(v, m, g-k_{x} ; c, n, g ;-k_{y}\right)=\Phi\left(v, m,-k_{x} ; c, n, 0 ;-k_{y}\right) \\
& =\int_{-\infty}^{\infty} \mathrm{d} y \varphi_{\mathrm{v}, m}^{*}\left(y-k_{x} l^{2}\right) \varphi_{\mathrm{c}, n}(y) \mathrm{e}^{-\mathrm{i} k_{y} y},
\end{aligned}
$$

where $n$ and $m$ are the numbers of Landau levels for conduction and valence electrons.
In the case $k_{x}=k_{y}=0$ we obtain from (49) the Kronecker delta symbol $\delta_{n m}$. It is the selection rule for the dipole-active optical transition, which takes place only when the number of Landau level of the electron in the valence band remains unchanged after its transition into the conduction band or, in other words, when the electron and hole of the pair created during the optical quantum transition have the same numbers of Landau levels $n=m$. The series expansion of the integrals (49) on the small parameter $\left(k_{x} \pm\right.$ $\left.\mathrm{i} k_{y}\right) l$ gives rise to multipole quantum transitions. The term in power zero determines the dipole-active transitions. The term in power 1 , i.e. proportional to $\left(k_{x} \pm \mathrm{i} k_{y}\right) l$, gives rise to the quadrupole-active optical quantum transitions with the selection rule $n=m \pm 1$ and probability proportional to $\left|\vec{k}_{\mathrm{D}} l\right|^{2} \approx(l / \lambda)^{2}$, where $\vec{k}_{\mathrm{D}}=k_{x} \vec{a}_{1}+k_{y} \vec{a}_{2}$ is the projection of the light wavevector $\vec{k}$ on the layer plane, $l$ is the magnetic length and $\lambda$ is the wavelength of the light.

These selection rules will be demonstrated below. Other matrix elements of the electron-radiation Hamiltonian (44) were calculated similarly. The optical quantum transitions were calculated between the initial state in the form of the ground state of the crystal in the presence of one circularly polarized photon with wavevector $\vec{Q}$, i.e.

$$
|i, \pm\rangle=\left(C_{\vec{Q}, \mp}\right)^{\dagger}|0\rangle .
$$

Here $|0\rangle$ is the vacuum state of the crystal and of the photon field. The final states coincide with the magnetoexciton states (40).

The probabilities of the four quantum transitions discussed above are the following:

$$
\begin{aligned}
& \left|\left\langle i, \mp\left|\hat{H}_{\mathrm{e}-\mathrm{rad}}\right| F_{1}\right\rangle\right|^{2} \\
& \quad=\left|a_{0}\right|^{2}\left|d_{0}\right|^{2}\left(\frac{e}{m_{0}}\right)^{2}\left|P_{\mathrm{vc}}\right|^{2} \frac{2 \pi \hbar N}{V \omega_{Q}}\left|\left(\vec{\sigma}_{\vec{Q}}^{\mp *} \cdot \vec{\sigma}_{-1}\right)\right|^{2} .
\end{aligned}
$$

Here $N=S /\left(2 \pi l^{2}\right)$ and $V=S L$. It is a dipole transition.

$\left|\left\langle i, \mp\left|\hat{H}_{\mathrm{e}-\mathrm{rad}}\right| F_{2}\right\rangle\right|^{2} \cong\left|\vec{Q}_{2 \mathrm{D}} l\right|^{6}\left|\left(\vec{\sigma}_{\vec{Q}}^{\mp *} \cdot \vec{\sigma}_{-1}\right)\right|^{2} \approx 0$.

It is a higher multipole transition and practically forbidden.

$$
\begin{aligned}
& \left|\left\langle i, \mp\left|\hat{H}_{\mathrm{e}-\mathrm{rad}}\right| F_{3}\right\rangle\right|^{2} \\
& \quad=\left|\vec{Q}_{2 \mathrm{D}} l\right|^{2}\left|b_{1}\right|^{2}\left(\frac{e}{m_{0}}\right)^{2}\left|P_{\mathrm{vc}}\right|^{2} \frac{2 \pi \hbar N}{V \omega_{Q}}\left|\left(\vec{\sigma}_{\vec{Q}}^{\mp *} \cdot \vec{\sigma}_{+1}\right)\right|^{2} .
\end{aligned}
$$

It is a weak quadrupole transition because $\left|b_{1}\right|^{2}<1$.

$$
\begin{aligned}
& \left|\left\langle i, \mp\left|\hat{H}_{\mathrm{e}-\mathrm{rad}}\right| F_{4}\right\rangle\right|^{2} \\
& \quad=\left(\frac{e}{m_{0}}\right)^{2}\left|P_{\mathrm{vc}}\right|^{2} \frac{2 \pi \hbar N}{V \omega_{Q}}\left|\left(\vec{\sigma}_{\vec{Q}}^{\mp *} \cdot \vec{\sigma}_{+1}\right)\right|^{2} .
\end{aligned}
$$

It is a dipole transition. $\vec{Q}_{2 \mathrm{D}}$ is the projection of the light wavevector $\vec{Q}$ on the layer plane.

We have calculated the creation energies, the matrix elements and the probabilities of the quantum transitions for eight combinations of the electron and hole Landau quantization states taking into account the RSOC.

The energies $E_{\mathrm{cv}}\left(F_{i}\right)$ of the band-to-band quantum transitions were calculated for all eight states. For the four 


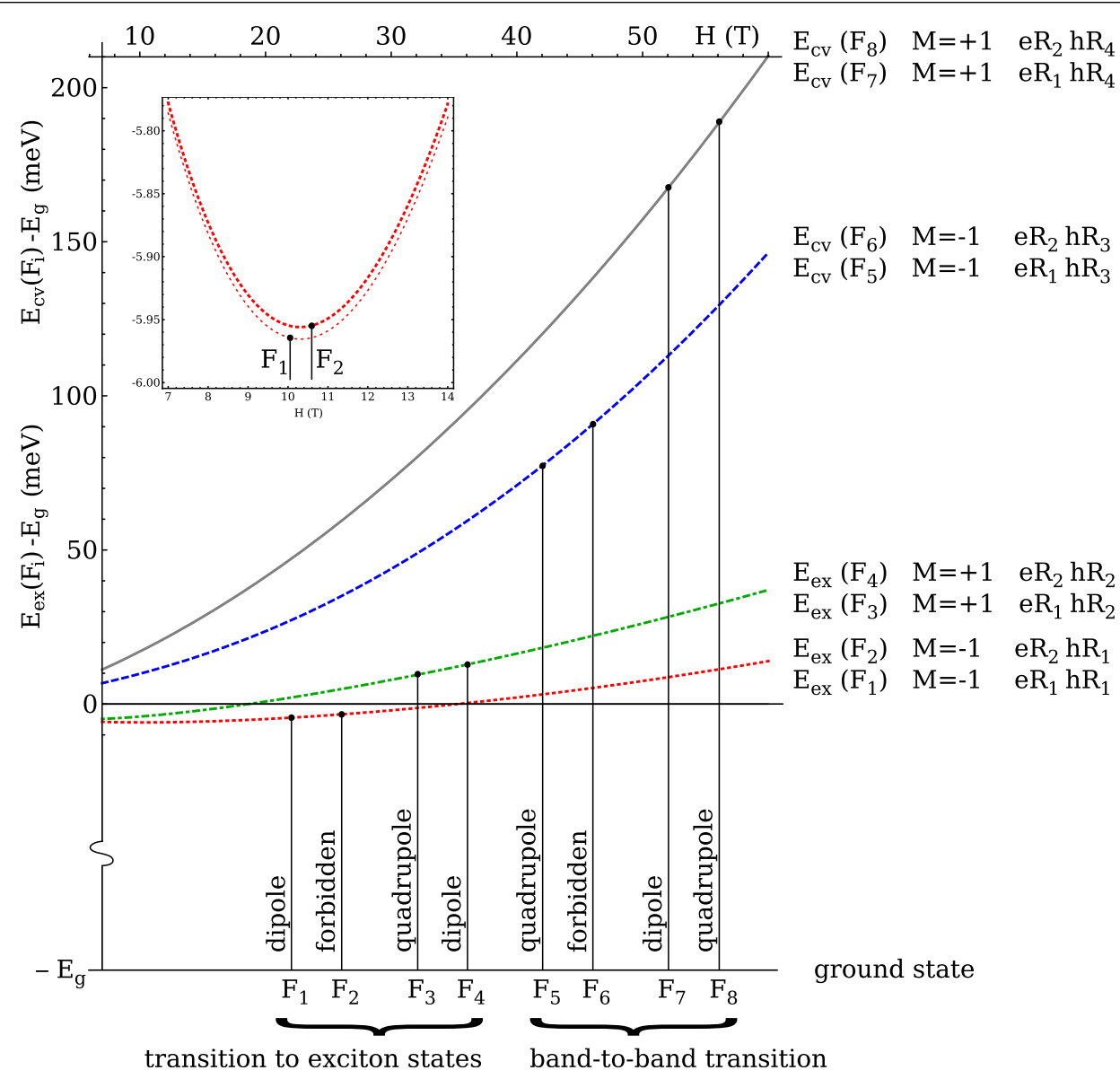

Figure 5. The quantum transitions from the ground state of the crystal to four magnetoexciton states, as well as for four band-to-band quantum transitions, involving two conduction electron states and four HH states taking into account the RSOC depending on the magnetic field strength.

combinations $F_{1}, F_{2}, F_{3}$ and $F_{4}$ the magnetoexciton binding energies were calculated also. This allows us to discuss the optical quantum transitions to the four lowest magnetoexciton levels at the point $k=0$. The first group of quantum transitions concerns the magnetoexcitons, whereas the second group $F_{5}$, $F_{6}, F_{7}$ and $F_{8}$ deals only with the band-to-band transitions. Between the eight states four of them, $F_{1}, F_{2}, F_{5}$ and $F_{6}$, are characterized by the $\mathrm{HH}$ orbital quantum number $M=$ -1 , whereas the other four states, $F_{3}, F_{4}, F_{7}$ and $F_{8}$, are characterized by the number $M=+1$.

Light with circular polarization $\vec{\sigma}_{\vec{Q}}^{ \pm}$will induce the quantum transitions in the states with a given number $M$ with the probabilities determined by the factors

$$
\left|\left(\vec{\sigma}_{\vec{Q}}^{ \pm *} \cdot \vec{\sigma}_{M}\right)\right|^{2}
$$

In the Faraday geometry when the light wavevector $\vec{Q}$ is oriented along the magnetic field direction the circular polarizations $\vec{\sigma}_{\vec{Q}}^{ \pm}$coincide with the exciton circular polarizations $\vec{\sigma}_{ \pm 1}$. In this case the light with circular polarization $\vec{\sigma}_{\vec{Q} \| \vec{H}}^{ \pm}$leads to the factor $\left(\vec{\sigma}_{\vec{Q}|| \vec{H}}^{ \pm *} \cdot \vec{\sigma}_{ \pm 1}\right)=\left(\vec{\sigma}_{ \pm 1}^{*}\right.$. $\left.\vec{\sigma}_{ \pm 1}\right)=1$ and excites the exciton states with the same polarizations $\vec{\sigma}_{ \pm 1}$. Such optical orientation of the exciton states under the influence of the circularly polarized light following [33] is called optical alignment.

There are three dipole-active optical quantum transitions in the states $F_{1}, F_{4}$ and $F_{7}$, two of them in the states $F_{1}$ and $F_{4}$ being strong, whereas the transition in the state $F_{7}$ is proportional to a small coefficient $\left|b_{1}\right|^{2}<1$.

Another three quantum transitions, $F_{3}, F_{5}$ and $F_{8}$, are quadrupole-active with probabilities proportional to a small factor $\left|\vec{Q}_{2 \mathrm{D}} l\right|^{2} \approx(l / \lambda)^{2}$, where $l$ is the magnetic length and $\lambda$ is the wavelength of the light. The existence of the quadrupole-active Wannier-Mott exciton states is a rare event in crystal optics, so is the case of the $1 \mathrm{~s}, \Gamma_{5}^{+}$, ortho-exciton state in $\mathrm{Cu}_{2} \mathrm{O}$ [32]. Such states in the case of magnetoexcitons were not revealed until now to the best of our knowledge. In addition one of the quadrupole transitions, $F_{3}$, has a supplementary weak factor $\left|b_{1}\right|^{2}<1$. The remaining two quantum transitions $F_{2}$ and $F_{6}$ are active only in the higher order of the multipole transitions, which means that they are practically forbidden. The described situation is represented in figure 5. The four curves represented here are doubled in reality with very small differences between them, as is demonstrated in the inset for the concrete states $F_{1}$ and $F_{2}$. The quantum transitions $F_{1}$ and $F_{2}$ take place on different curves of this doublet. 


\section{Conclusions}

The influence of the RSOC on the properties of the 2D magnetoexcitons was determined. The interdependence between the Landau quantization of the electron and hole orbital motions and their spin projections was revealed in the frame of the Landau gauge and the secondary quantization description. The spinor-type wavefunctions (38) of the 2D conduction and valence electrons in the presence of the RSOC have different numbers of Landau quantization functions for different spin projections. For example, they are $\varphi_{0}(y)$ and $\varphi_{1}(y)$ in one case, and $\varphi_{0}(y)$ and $\varphi_{3}(y)$ in another one. For a conduction electron, following the solution (38), if the number of Landau level is $n$ for the up spin projection, it is equal to $n+1$ for the down spin projection. For a valence electron the number $n$ of the Landau level for the up spin projection is accompanied by the number $n+3$ for the down spin projection. It is determined completely by the fact that the RSOC Hamiltonian for a conduction electron is linear in the projections $k_{ \pm}=k_{x} \pm \mathrm{i} k_{y}$ of the in-plane wavevector $\vec{k}_{\|}$, whereas in the case of a valence electron and an $\mathrm{HH}$ the corresponding Hamiltonian contains the third order of these projections, i.e. the expressions $\left(k_{ \pm}\right)^{3}$. The shifts and the rearrangements of the $2 \mathrm{D}$ hole Landau quantization levels on the energy scale are much larger in comparison with the case of the conduction electron Landau levels. This is due to the strong influence of the magnetic field strength on the RSOC parameter. At some values of this parameter the shifts and rearrangements are comparable with the hole cyclotron energy. The two LLLs $R_{1}$ and $R_{2}$ for a conduction electron and the four lowest hole states $R_{1}, R_{2}, R_{3}$ and $R_{4}$ were considered. The wavefunctions (38) were used to calculate the matrix elements of the Coulomb direct $\mathrm{e}-\mathrm{h}$ interactions corresponding to the combinations $F_{1}=\left(e, R_{1} ; h, R_{1}\right) ; F_{2}=$ $\left(e, R_{2} ; h, R_{1}\right) ; F_{3}=\left(e, R_{1} ; h, R_{2}\right) ; F_{4}=\left(e, R_{2} ; h, R_{2}\right) ; F_{5}=$ $\left(e, R_{1} ; h, R_{3}\right) ; F_{6}=\left(e, R_{2} ; h, R_{3}\right) ; F_{7}=\left(e, R_{1} ; h, R_{4}\right)$ and $F_{8}=\left(e, R_{2} ; h, R_{4}\right)$. The corresponding ionization potentials were expressed through the ionization potentials of the bare magnetoexciton states $I_{\mathrm{ex}}^{(n, m)}(k)$ calculated earlier in [30]. With their help as well as with the knowledge of the coefficients $a_{0}$, $b_{1}, d_{0}, c_{3}$ (27), (34), and so on, it was possible to determine the dispersion laws (42), (43) of the eight new magnetoexciton bands taking into account the RSOC. The new dispersion laws could lead to new collective properties of the spinor-type 2D magnetoexcitons.

The optical quantum transitions from the ground state of the crystal to four magnetoexciton states were determined on the basis of the exciton wavefunctions (40) and electron-radiation interaction (44). It was shown that there are three dipole-active quantum transitions in the states $F_{1}$, $F_{4}$ and $F_{7}$ with exciton alignments and polarizations $\vec{\sigma}_{-1}$, $\vec{\sigma}_{1}$ and $\vec{\sigma}_{1}$ correspondingly, three quadrupole-active quantum transitions $F_{3}, F_{5}$ and $F_{8}$ with polarizations $\vec{\sigma}_{1}, \vec{\sigma}_{-1}$ and $\vec{\sigma}_{1}$ correspondingly and two forbidden quantum transitions $F_{2}$ and $F_{6}$. The alignment appears due to the factors $\mid\left(\vec{\sigma}_{\vec{Q}}^{ \pm *}\right.$. $\left.\vec{\sigma}_{ \pm 1}\right)\left.\right|^{2}$, which depend on the scalar products between the vectors of the light circular polarizations arbitrarily oriented in space and the vectors of the circular polarizations $\vec{\sigma}_{ \pm 1}$ of the HHs and of the magnetoexcitons as a whole, oriented perpendicular to the layer along the magnetic field strength. In the Faraday geometry when the light wavevector $\vec{Q}$ is parallel to the magnetic field direction, the vectors of the light circular polarization $\vec{\sigma}_{\vec{Q} \| \vec{H}}^{ \pm}$coincide with the vectors of the hole circular polarization $\vec{\sigma}_{ \pm 1}$, the dipole quantum transitions transfer the polarizations from the light to the matter, whereas the quadrupole quantum transitions vanish because the projection $\vec{Q}_{2 \mathrm{D}}$ of the light wavevector $\vec{Q}$ on the layer surface equals zero.

\section{Acknowledgment}

IVP gratefully acknowledges the Foundation for Young Scientists of the Academy of Sciences of Moldova for financial support (11.819.05.13F).

\section{References}

[1] Rashba E I 1960 Fiz. Tverd. Tela 21224

[2] Hakioğlu T and Şahin M 2007 Phys. Rev. Lett. 98166405

[3] Ali Can M and Hakioğlu T 2009 Phys. Rev. Lett. 103086404

[4] Dresselhaus G 1955 Phys. Rev. 100580

[5] Bychkov Yu A and Rashba E I 1984 Pis. Zh. Eksp. Teor. Fiz. 3966

[6] Winkler R 2003 Spin-Orbit Coupling Effects in Two-Dimensional Electron and Hole Systems (Springer Tracts in Modern Physics vol 191) (Berlin: Springer)

[7] Zhu B-f and Chang Y-C 1994 Phys. Rev. B 5011932

[8] van Gelderen R and Smith C M 1994 Phys. Rev. B 81125435

[9] Brazovskii S A 1975 Zh. Eksp. Teor. Fiz. 68175 Brazovskii S A 1975 Sov. Phys._JETP 4185 (Engl. transl.)

[10] Winkler R, Merkler M, Darnhofer T and Rossler U 1996 Phys. Rev. B $\mathbf{5 3} 10858$

[11] Ekenberg U and Altarelli M 1985 Phys. Rev. B 323712

[12] Winkler R 2000 Phys. Rev. B 624245

[13] Winkler R, Noh H, Tutuc E and Shayegan M 2002 Phys. Rev. B 65155303

[14] Ivchenko E L and Pikus G E 1997 Superlattices and Other Heterostructures. Symmetry and Optical Phenomena (Springer Series in Solid-State Sciences vol 110) (Berlin: Springer) p 370

[15] Castro Neto A H, Guinea F, Peres N M R, Novoselov K S and Geim A K 2009 Rev. Mod. Phys. 81109

[16] Miller D L, Kubista K D, Rutter G M, Ruan M, de Heer W A, First P N and Stroscio J A 2009 Science 324924

[17] Peres N M R and Castro Neto A H 2007 J. Phys.: Condens. Matter 19406231

[18] Guinea F, Castro Neto A H and Peres N M R 2006 Phys. Rev. B 73245426

[19] Milton Pereira J Jr, Peeters F M and Vasilopoulos P 2007 Phys. Rev. B 76115419

[20] Novoselov K S, McCann E, Morozov S V, Falko V I, Katsnelson M I, Zeitler U, Jiang D, Schedin F and Geim A K 2006 Nature Phys. 2177

[21] Bir G L and Pikus G E 1974 Symmetry and Strain-Induced Effects in Semiconductors (New York: Wiley)

[22] Schliemann J and Loss D 2005 Phys. Rev. B 71085308

[23] Moskalenko S A and Snoke D W 2000 Bose-Einstein Condensation of Excitons and Biexcitons and Coherent Nonlinear Optics with Excitons (Cambridge: Cambridge University Press) p 415

[24] Das B, Datta S and Reifenberg R 1990 Phys. Rev. B 418278 
[25] Falko V I 1992 Phys. Rev. B 464320

Falko V I 1993 Phys. Rev. Lett. 71141

[26] Schliemann J, Egues J C and Loss D 2003 Phys. Rev. B 67085302

[27] Winkler R, Zulicke U and Bolte J 2007 Phys. Rev. B 75205314

[28] Schliemann J 2008 Phys. Rev. B 77125303

[29] Moskalenko S A, Khadzhi P I, Podlesny I V, Novikov B V and Kiselyov A A 2011 Solid State Commun. accepted
[30] Moskalenko S A, Liberman M A, Khadzhi P I, Dumanov E V, Podlesny Ig V and Boţan V V 2007 Physica E 39137

[31] Moskalenko S A, Liberman M A and Podlesny I V 2009 Phys. Rev. B 79125425

[32] Moskalenko S A 1983 Introduction in the Theory of High Density Excitons (Chisinau: Shtiintsa) (in Russian)

[33] Meier F and Zakharchenya B P (ed) 1984 Optical Orientation (Modern Problems in Condensed Matter Sciences vol 8) (Amsterdam: North-Holland) 